This is the author's final, peer-reviewed manuscript as accepted for publication. The publisher-formatted version may be available through the publisher's web site or your institution's library.

\title{
Hubble parameter measurement constraints on dark energy
}

Omer Farooq, Data Mania, and Bharat Ratra

\section{How to cite this manuscript}

If you make reference to this version of the manuscript, use the following information:

Farooq, O., Mania, D., \& Ratra, B. (2013). Hubble parameter measurement constraints on dark energy. Retrieved from http://krex.ksu.edu

\section{Published Version Information}

Citation: Farooq, O., Mania, D., \& Ratra, B. (2013). Hubble parameter measurement constraints on dark energy. Astrophysical Journal, 764(2), 138.

Copyright: @ 2013. The American Astronomical Society

Digital Object Identifier (DOI): doi:10.1088/0004-637X/764/2/138

Publisher's Link: http://iopscience.iop.org/0004-637X/764/2/138/

This item was retrieved from the K-State Research Exchange (K-REx), the institutional repository of Kansas State University. K-REx is available at http://krex.ksu.edu 
KSUPT-12/5 November 2012

\title{
Hubble parameter measurement constraints on dark energy
}

\author{
Omer Farooq $^{1}$, Data Mania ${ }^{1,2}$, and Bharat Ratra ${ }^{1}$
}

\begin{abstract}
We use 21 Hubble parameter versus redshift data points, from Simon et al. (2005), Gaztañaga et al. (2009), Stern et al. (2010), and Moresco et al. (2012a), to place constraints on model parameters of constant and time-evolving dark energy cosmologies. The inclusion of the 8 new Moresco et al. (2012a) measurements results in $H(z)$ constraints more restrictive than those derived by Chen \& Ratra (2011b). These constraints are now almost as restrictive as those that follow from current Type Ia supernova (SNIa) apparent magnitude versus redshift data (Suzuki et al. 2012), which now more carefully account for systematic uncertainties. This is a remarkable result. We emphasize however that SNIa data have been studied for a longer time than the $H(z)$ data, possibly resulting in a better estimate of potential systematic errors in the SNIa case. A joint analysis of the $H(z)$, baryon acoustic oscillation peak length scale, and SNIa data favors a spatially-flat cosmological model currently dominated by a time-independent cosmological constant but does not exclude slowly-evolving dark energy.
\end{abstract}

\section{Introduction}

The expansion rate of the Universe changes with time, initially slowing when matter dominated, because of the mutual gravitational attraction of all the matter in it, and more recently accelerating. A number of cosmological observations now strongly support the idea that the Universe is spatially flat (provided the dark energy density is close to or time independent) and is currently undergoing accelerated cosmological expansion. A majority of cosmologists consider dark energy to be the cause of this observed accelerated cosmological

\footnotetext{
${ }^{1}$ Department of Physics, Kansas State University, 116 Cardwell Hall, Manhattan, KS 66506, USA omer@phys.ksu.edu,mania@phys.ksu.edu, and ratra@phys.ksu.edu

${ }^{2}$ Center for Elementary Particle Physics, Ilia State University, 3-5 Cholokashvili Ave., Tbilisi 0179, Georgia
} 
expansion. $^{1}$ This dark energy, most simply thought of as a negative pressure substance, dominates the current cosmological energy budget. For reviews of dark energy see Bass (2011), Jimenez (2011), Li et al. (2011a), Bolotin et al. (2011), and references therein.

Three observational techniques provide the strongest evidence for dark energy: SNIa apparent magnitude measurements as a function of redshift (e.g., Sullivan et al. 2011; Suzuki et al. 2012; Li et al. 2011b; Barreira \& Avelino 2011); cosmic microwave background (CMB) anisotropy data (Podariu et al. 2001b; Komatsu et al. 2011, and references therein) combined with low estimates of the cosmological mass density (e.g., Chen \& Ratra 2003), provided the dark energy density is close to or time independent; and baryon acoustic oscillation (BAO) peak length scale measurements (e.g., Beutler et al. 2011; Blake et al. 2011; Mehta et al. 2012).

The "standard" model of cosmology is the spatially-flat $\Lambda$ CDM model (Peebles 1984). In this model about $73 \%$ of the current energy budget is dark energy, Einstein's cosmological constant $\Lambda$. Non-relativistic cold dark matter (CDM) is the next largest contributer to the energy budget (around 23\%), followed by non-relativistic baryonic matter (about 5\%). For reviews of the standard model of cosmology see Ratra \& Vogeley (2008) and references therein. It has been known for a while that the $\Lambda$ CDM model is reasonably consistent with most observations (see, e.g., Jassal et al. 2010; Wilson et al. 2006; Davis et al. 2007; Allen et al. 2008, for early indications). ${ }^{2}$ In the $\Lambda$ CDM model the dark energy density is constant in time and does not vary in space.

Although most predictions of the $\Lambda$ CDM model are reasonably consistent with the measurements, the $\Lambda \mathrm{CDM}$ model has some curious features. For instance, the measured cosmological constant energy density is 120 orders of magnitude smaller than the energy density naively expected from quantum field theory (this is known as the fine-tuning puzzle). A second curiosity is what is known as the coincidence puzzle: the energy density of a cosmological constant, $\rho_{\Lambda}$, is independent of time, but that of matter, $\rho_{\mathrm{m}}$, decreases with time during the cosmological expansion, so it is curious why we (observers) happen to live at this (apparently) special time, when the dark energy and the non-relativistic matter energy densities are of comparable magnitude.

\footnotetext{
${ }^{1}$ Some cosmologists instead view these observations as an indication that general relativity needs to be modified on these large length scales. For recent reviews of modified gravity see Tsujikawa (2010), Bolotin et al. (2011), Capozziello \& De Laurentis (2011), Starkman (2011), and references therein. In this paper we assume that general relativity provides an adequate description of gravitation on cosmological scales.

${ }^{2}$ Note, however, that the "standard" CDM structure formation model, which is assumed in the $\Lambda$ CDM model, might need modification (see Peebles \& Ratra 2003; Perivolaropoulos 2010, and references therein).
} 
These puzzles could be partially resolved if the dark energy density is a slowly decreasing function of time (Peebles \& Ratra 1988; Ratra \& Peebles 1988). In this case the dark energy density will remain comparable to the non-relativistic matter density for a longer time. For recent discussions of time-varying dark energy models, see Bauer et al. (2011), Chimento et al. (2011), Granda et al. (2011), García-Bellido et al. (2011), Basilakos et al. (2012), Sheykhi et al. (2012), Brax \& Davis (2012), Hollenstein et al. (2012), Cai et al. (2012), and references therein. In this paper we will consider two dark energy models (with dark energy being either a cosmological constant or a slowly-evolving scalar field $\phi$ ) as well as a dark energy parameterization.

In the $\Lambda$ CDM model, time-independent dark energy density (the cosmological constant $\Lambda)$ is modeled as a spatially homogeneous fluid with equation of state $p_{\Lambda}=-\rho_{\Lambda}$. Here $p_{\Lambda}$ and $\rho_{\Lambda}$ are the fluid pressure and energy density. In describing slowly-decreasing dark energy density much use has been made of a parameterization known as XCDM. Here dark energy is modeled as a spatially homogeneous $X$-fluid with equation of state $p_{\mathrm{X}}=w_{\mathrm{X}} \rho_{\mathrm{X}}$. The equation of state parameter $w_{\mathrm{X}}<-1 / 3$ is independent of time and $p_{\mathrm{X}}$ and $\rho_{\mathrm{X}}$ are the pressure and energy density of the $X$-fluid. When $w_{\mathrm{X}}=-1$ the XCDM parameterization reduces to the complete and consistent $\Lambda$ CDM model. For any other value of $w_{\mathrm{X}}<-1 / 3$ the XCDM parameterization is incomplete as it cannot describe spatial inhomogeneities (see, e.g. Ratra 1991; Podariu \& Ratra 2000). For computational simplicity, in the XCDM case we assume a spatially-flat cosmological model.

The $\phi \mathrm{CDM}$ model is the simplest, consistent and complete model of slowly-decreasing dark energy density (Peebles \& Ratra 1988; Ratra \& Peebles 1988). In this model dark energy is modeled as a scalar field, $\phi$, with a gradually decreasing (in $\phi$ ) potential energy density $V(\phi)$. Here we assume an inverse power-law potential energy density $V(\phi) \propto \phi^{-\alpha}$, where $\alpha$ is a nonnegative constant (Peebles \& Ratra 1988). When $\alpha=0$ the $\phi$ CDM model reduces to the corresponding $\Lambda \mathrm{CDM}$ case. For computational simplicity, we again only consider the spatially-flat cosmological case for $\phi \mathrm{CDM}$.

As mentioned above, for some time now, most observational constraints have been reasonably consistent with the predictions of the "standard" spatially-flat $\Lambda$ CDM model. CMB anisotropy, SNIa, and BAO measurements provide the strongest support for this conclusion. However, the error bars associated with these three types of data are still too large to allow for a significant observational discrimination between the $\Lambda$ CDM model and the two simple time-varying dark energy models discussed above. This is one motivation for considering additional kinds of data.

If the constraints from the new data differ considerably from the old ones, this could mean that at least one of the data sets had an undetected systematic error, or it could mean 
that the model being tested is observationally inconsistent. Either of these is an important result. On the other hand, if the constraints from the new and the old data are consistent, then a joint analysis of all the data could result in tighter constraints, and so might result in significantly discriminating between constant and time-varying dark energy models.

Other measurements that have been used to constrain cosmological parameters ${ }^{3}$ include galaxy cluster gas mass fraction as a function of redshift (e.g., Allen et al. 2008; Samushia \& Ratra 2008; Ettori et al. 2009; Tong \& Noh 2011; Lu et al. 2011), galaxy cluster and other large-scale structure properties (Campanelli et al. 2012; De Boni et al. 2011; Mortonson et al. 2011; Devi et al. 2011; Wang 2012, and references therein), gamma-ray burst luminosity distance as a function of redshift (e.g., Samushia \& Ratra 2010; Wang \& Dai 2011; Busti et al. 2012), lookback time as a function of redshift (Samushia et al. 2010; Dantas et al. 2011; Tonoiu et al. 2011, and references therein), HII starburst galaxy apparent magnitude as a function of redshift (e.g., Plionis et al. 2010, 2011; Mania \& Ratra 2012), angular size as a function of redshift (e.g., Guerra et al. 2000; Bonamente et al. 2006; Chen \& Ratra 2012), and strong gravitational lensing (Chae et al. 2004; Lee \& Ng 2007; Biesiada et al. 2010; Zhang \& Wu 2010, and references therein). ${ }^{4}$ Of particular interest to us here are measurements of the Hubble parameter as a function of redshift (e.g., Jimenez et al. 2003; Samushia \& Ratra 2006; Samushia et al. 2007; Sen \& Scherrer 2008; Pan et al. 2010; Chen \& Ratra 2011b; Kumar 2012; Wang \& Zhang 2011; Duan et al. 2011; Bilicki et al. 2012; Seikel et al. 2012). While the constraints from these data are typically less restrictive than those derived from the SNIa, CMB anisotropy, and BAO data, both types of measurements result in largely compatible constraints that generally support a currently accelerating cosmological expansion. This provides confidence that the broad outlines of a "standard" cosmological model are now in place.

In this paper we use the $21 H(z)$ measurements of Simon et al. (2005), Gaztañaga et al. (2009), Stern et al. (2010), and Moresco et al. (2012a) (listed in Table 1$)^{5}$ to constrain the $\Lambda \mathrm{CDM}$ and $\phi \mathrm{CDM}$ models and the XCDM parametrization. The inclusion of the 8 new Moresco et al. (2012a) measurements (with smaller error bars compared to the earlier data) in the analysis results in tighter constraints than those recently derived by Chen \& Ratra

\footnotetext{
${ }^{3}$ For reviews see Albrecht et al. (2006), Weinberg et al. (2012), and references therein.

${ }^{4}$ Future space-based SNIa and BAO-like meassurements (e.g., Podariu et al. 2001a; Samushia et al. 2011; Sartoris et al. 2012; Basse et al. 2012; Pavlov et al. 2012), as well as measurements based on new techniques (Jennings et al. 2011; van de Weygaert et al. 2011; Ziaeepour 2012, and references therein) should soon provide interesting constraints on cosmological parameters.

${ }^{5}$ We do not include the 4 recent Zhang et al. (2012) $H(z)$ measurements as they have somewhat larger error bars and do not affect our results.
} 


\begin{tabular}{cccc}
\hline \hline$z$ & $\begin{array}{c}H(z) \\
\left(\mathrm{km} \mathrm{s}^{-1} \mathrm{Mpc}^{-1}\right)\end{array}$ & $\begin{array}{c}\sigma_{H} \\
\left(\mathrm{~km} \mathrm{~s}^{-1} \mathrm{Mpc}^{-1}\right)\end{array}$ & Reference \\
\hline 0.090 & 69 & 12 & 1 \\
0.170 & 83 & 8 & 1 \\
0.179 & 75 & 4 & 4 \\
0.199 & 75 & 5 & 4 \\
0.240 & 79.69 & 2.65 & 2 \\
0.270 & 77 & 14 & 1 \\
0.352 & 83 & 14 & 4 \\
0.400 & 95 & 17 & 1 \\
0.430 & 86.45 & 3.68 & 2 \\
0.480 & 97 & 62 & 3 \\
0.593 & 104 & 13 & 4 \\
0.680 & 92 & 8 & 4 \\
0.781 & 105 & 12 & 4 \\
0.875 & 125 & 17 & 4 \\
0.880 & 90 & 40 & 3 \\
0.900 & 117 & 23 & 1 \\
1.037 & 154 & 20 & 4 \\
1.300 & 168 & 17 & 1 \\
1.430 & 177 & 18 & 1 \\
1.530 & 140 & 14 & 1 \\
1.750 & 202 & 40 & 1 \\
\hline \hline
\end{tabular}

Table 1: Hubble parameter versus redshift data. Last column reference numbers: 1. Simon et al. (2005), 2. Gaztañaga et al. (2009), 3. Stern et al. (2010), 4. Moresco et al. (2012a). 
(2011b) from the previous largest set of $H(z)$ measurements considered. The new $H(z)$ data constraints derived here are compatible with cosmological parameter constraints determined by other techniques. For the first time, these $H(z)$ limits are almost as constraining as those derived from the most recent SNIa data compilation of Suzuki et al. (2012). In addition to the tighter $H(z)$ limits resulting from the new data, this is partially also a consequence of the fact that a more careful analysis of the SNIa measurements (Suzuki et al. 2012) has resulted in a larger systematic error estimate and thus weaker SNIa constraints. We emphasize that the study of $H(z)$ data is much less mature than that of SNIa apparent magnitude data, so there is the possibility that future $H(z)$ error bars might be larger than what we have used in our analysis here. In addition to deriving $H(z)$-data only constraints, we also use these $H(z)$ data in combination with recent BAO and SNIa measurements to jointly constrain cosmological parameters in these models. ${ }^{6}$ Adding the $H(z)$ data tightens the constraints, somewhat significantly in some parts of parameter space for some of the models we study.

Our paper is organized as follows. In Sec. 2 we present the basic equations of the three dark energy models we consider. Constraints from the $H(z)$ data are derived in Sec. 3. In Sec. 4 we determine constraints from recent SNIa apparent magnitude data. In Sec. 5 we derive constraints from recent BAO data. Joint constraints on cosmological parameters, from a combined analysis of the three data sets, for the three models we consider, are presented in Sec. 6. We conclude in Sec. 7.

\section{Dark energy models}

In this section we summarize properties of the two models $(\Lambda \mathrm{CDM}$ and $\phi \mathrm{CDM})$ and the one parametrization (XCDM) we use in our analyses of the data.

To determine how the Hubble parameter $H(z)$ evolves in these models, we start from the Einstein equation of general relativity

$$
R_{\mu \nu}-\frac{1}{2} g_{\mu \nu} R=8 \pi G T_{\mu \nu}-\Lambda g_{\mu \nu}
$$

Here $g_{\mu \nu}$ is the metric tensor, $R_{\mu \nu}$ and $R$ are the Ricci tensor and scalar, $T_{\mu \nu}$ is the energymomentum tensor of any matter present, $\Lambda$ is the cosmological constant, and $G$ is the Newtonian gravitational constant.

The energy-momentum tensor for an ideal fluid is $T_{\mu \nu}=\operatorname{diag}(\rho, p, p, p)$, where $\rho$ is the

\footnotetext{
${ }^{6}$ See Moresco et al. (2012b) and Wang et al. (2012) for analyses that use most of these $H(z)$ data in conjunction with CMB anisotropy and other data to constrain cosmological parameters.
} 
energy density and $p$ the pressure. Assuming spatial homogeneity, the Einstein equation reduces to the two independent Friedmann equations

$$
\begin{aligned}
& \left(\frac{\dot{a}}{a}\right)^{2}=\frac{8 \pi G}{3} \rho+\frac{\Lambda}{3}-\frac{K^{2}}{a^{2}}, \\
& \frac{\ddot{a}}{a}=-\frac{4 \pi G}{3}(\rho+3 p)+\frac{\Lambda}{3} .
\end{aligned}
$$

Here $a(t)$ is the cosmological scale factor, an overdot denotes a derivative with respect to time, and $K^{2}$ represents the curvature of the spatial hypersurfaces. These equations, in conjunction with the equation of state,

$$
p=p(\rho)=\omega \rho,
$$

where $\omega$ is the dimensionless equation-of-state parameter (with $\omega=-1$ corresponding to a cosmological constant and $\omega<-1 / 3$ corresponding to the XCDM parametrization), govern the evolution of the scale factor and matter densities.

Taking the time derivative of Eq. (2) and putting it in Eq. (3) and then using Eq. (4) yields the energy conservation equation

$$
\dot{\rho}=-3 \frac{\dot{a}}{a}(\rho+p)=-3 \rho \frac{\dot{a}}{a}(1+\omega)
$$

For a non-relativistic gas (matter) $\omega=\omega_{m}=0$ and $\rho_{m} \propto a^{-3}$, and for a cosmological constant $\omega=\omega_{\Lambda}=-1$ and $\rho_{\Lambda}=\Lambda /(8 \pi G)=$ constant $\left(\dot{\rho_{\Lambda}}=0\right)$. Solving Eq. (5), the time-dependent energy density is

$$
\rho(t)=\rho_{0}\left(\frac{a_{0}}{a}\right)^{3(1+\omega)}
$$

where $\rho_{0}$ and $a_{0}$ are the current values of the fluid energy density and the scale factor. If there are a number of different species of non-interacting particles, then Eq. (6) holds separately for each of them.

The ratio $\dot{a}(t) / a(t)=H(t)$ is called the Hubble parameter. The present value of the Hubble parameter is known as the Hubble constant and is denoted by $H_{0}$. Defining the redshift $z=a_{0} / a-1$, and the present value of the density parameters,

$$
\Omega_{m 0}=\frac{8 \pi G \rho_{0}}{3 H_{0}^{2}}, \quad \Omega_{K 0}=\frac{-K^{2}}{\left(H_{0} a_{0}\right)^{2}}, \quad \Omega_{\Lambda}=\frac{\Lambda}{3 H_{0}^{2}},
$$

in the $\Lambda$ CDM model we can rewrite Eq. (2) as

$$
H^{2}\left(z ; H_{0}, \mathbf{p}\right)=H_{0}^{2}\left[\Omega_{m 0}(1+z)^{3}+\Omega_{\Lambda}+\left(1-\Omega_{m 0}-\Omega_{\Lambda}\right)(1+z)^{2}\right]
$$


where we have made use of $\Omega_{K 0}=1-\Omega_{m 0}-\Omega_{\Lambda}$. This is the Friedmann equation of the $\Lambda$ CDM model with spatial curvature. In this model the cosmological parameters $\mathbf{p}=\left(\Omega_{m 0}, \Omega_{\Lambda}\right)$. Here $\Omega_{m 0}$ is the non-relativistic (baryonic and cold dark) matter energy density parameter at the present time. Below we shall have need for the dimensionless Hubble parameter $E(z)=H(z) / H_{0}$.

It has become popular to parametrize time-varying dark energy as a spatially homogeneous $X$-fluid, with a constant equation of state parameter $\omega_{\mathrm{X}}=p_{\mathrm{X}} / \rho_{\mathrm{X}}<-1 / 3$. With this XCDM parametrization the Friedmann equation takes the form

$$
H^{2}\left(z ; H_{0}, \mathbf{p}\right)=H_{0}^{2}\left[\Omega_{m 0}(1+z)^{3}+\left(1-\Omega_{m 0}\right)(1+z)^{3\left(1+\omega_{\mathrm{X}}\right)}\right]
$$

where for computational simplicity we consider only flat spatial hypersurfaces, and the model parameters $\mathbf{p}=\left(\Omega_{m 0}, \omega_{\mathrm{X}}\right)$. The XCDM parametrization is incomplete, as it cannot describe the evolution of energy density inhomogeneities.

The simplest complete and consistent dynamical dark energy model is $\phi$ CDM. In this model dark energy is a slowly-rolling scalar field $\phi$ with an, e.g., inverse-power-law potential energy density $V(\phi)=\kappa m_{p}^{2} \phi^{-\alpha}$ where $m_{p}=1 / \sqrt{G}$ is the Planck mass and $\alpha$ is a nonnegative free parameter that determines $\kappa$. The scalar field part of the $\phi$ CDM model action is

$$
S=\frac{m_{p}^{2}}{16 \pi} \int \sqrt{-g}\left(\frac{1}{2} g^{\mu \nu} \partial_{\mu} \phi \partial_{\nu} \phi-\kappa m_{p}^{2} \phi^{-\alpha}\right) d^{4} x,
$$

with corresponding scalar field equation of motion

$$
\ddot{\phi}+3 \frac{\dot{a}}{a} \dot{\phi}-\kappa \alpha m_{p}^{2} \phi^{-(\alpha+1)}=0 .
$$

In the spatially-flat case the Friedmann equation is

$$
H^{2}\left(z ; H_{0}, \mathbf{p}\right)=\frac{8 \pi G}{3}\left(\rho_{m}+\rho_{\phi}\right)=H_{0}^{2}\left[\Omega_{m 0}(1+z)^{3}+\Omega_{\phi}(z, \alpha)\right],
$$

with scalar field energy density given by

$$
\rho_{\phi}=\frac{m_{p}^{2}}{16 \pi}\left(\frac{1}{2} \dot{\phi}^{2}+\kappa m_{p}^{2} \phi^{-\alpha}\right) .
$$

Solving the coupled differential Eqs. (11) - (13), with the initial conditions described in Peebles \& Ratra (1988), allows for a numerical computation of the Hubble parameter $H(z)$. In this case the model parameter set is $\mathbf{p}=\left(\Omega_{m 0}, \alpha\right)$. 


\section{Constraints from the $H(z)$ data}

We use 21 independent $H(z)$ data points (Simon et al. 2005; Gaztañaga et al. 2009; Stern et al. 2010; Moresco et al. 2012a), listed in Table 1, to constrain cosmological model parameters. The observational data consist of measurements of the Hubble parameter $H_{\text {obs }}\left(z_{i}\right)$ at redshifts $z_{i}$, with the corresponding one standard deviation uncertainties $\sigma_{i}$.

To constrain cosmological parameters $\mathbf{p}$ of the models of interest we compute the $\chi_{H}^{2}$ function

$$
\chi_{H}^{2}\left(H_{0}, \mathbf{p}\right)=\sum_{i=1}^{21} \frac{\left[H_{\mathrm{th}}\left(z_{i} ; H_{0}, \mathbf{p}\right)-H_{\mathrm{obs}}\left(z_{i}\right)\right]^{2}}{\sigma_{i}^{2}} .
$$

where $H_{\mathrm{th}}\left(z_{i} ; H_{0}, \mathbf{p}\right)$ is the model-predicted value of the Hubble parameter. As discussed in Sec. $2, H_{\mathrm{th}}\left(z_{i} ; H_{0}, \mathbf{p}\right)=H_{0} E(z ; \mathbf{p})$, so from Eq. (14) we find

$$
\chi_{H}^{2}\left(H_{0}, \mathbf{p}\right)=H_{0}^{2} \sum_{i=1}^{21} \frac{E^{2}\left(z_{i} ; \mathbf{p}\right)}{\sigma_{i}^{2}}-2 H_{0} \sum_{i=1}^{21} \frac{H_{\mathrm{obs}}\left(z_{i}\right) E\left(z_{i} ; \mathbf{p}\right)}{\sigma_{i}^{2}}+\sum_{i=1}^{21} \frac{H_{\mathrm{obs}}^{2}\left(z_{i}\right)}{\sigma_{i}^{2}} .
$$

$\chi_{H}^{2}$ depends on the model parameters $\mathbf{p}$ as well as on the nuisance parameter $H_{0}$ whose value is not known exactly. We assume that the distribution of $H_{0}$ is a Gaussian with one standard deviation width $\sigma_{H_{0}}$ and mean $\bar{H}_{0}$. We can then build the posterior likelihood function $\mathcal{L}_{H}(\mathbf{p})$ that depends only on the $\mathbf{p}$ by integrating the product of $\exp \left(-\chi_{H}^{2} / 2\right)$ and the $H_{0}$ prior likelihood function $\exp \left[-\left(H_{0}-\bar{H}_{0}\right)^{2} /\left(2 \sigma_{H_{0}}^{2}\right)\right]$ (see, e.g., Ganga et al. 1997),

$$
\mathcal{L}_{H}(\mathbf{p})=\frac{1}{\sqrt{2 \pi \sigma_{H_{0}}^{2}}} \int_{0}^{\infty} e^{-\chi_{H}^{2}\left(H_{0}, \mathbf{p}\right) / 2} e^{-\left(H_{0}-\bar{H}_{0}\right)^{2} /\left(2 \sigma_{H_{0}}^{2}\right)} d H_{0}
$$

Defining

$$
\alpha=\frac{1}{\sigma_{H_{0}}^{2}}+\sum_{i=1}^{21} \frac{E^{2}\left(z_{i} ; \mathbf{p}\right)}{\sigma_{i}^{2}}, \beta=\frac{\bar{H}_{0}}{\sigma_{H_{0}}^{2}}+\sum_{i=1}^{21} \frac{H_{\mathrm{obs}}\left(z_{i}\right) E\left(z_{i} ; \mathbf{p}\right)}{\sigma_{i}^{2}}, \gamma=\frac{\bar{H}_{0}^{2}}{\sigma_{H_{0}}^{2}}+\sum_{i=1}^{21} \frac{H_{\mathrm{obs}}^{2}\left(z_{i}\right)}{\sigma_{i}^{2}}
$$

the integral can be expressed in terms of the error function, ${ }^{7}$

$$
\mathcal{L}_{H}(\mathbf{p})=\frac{1}{2 \sqrt{\alpha \sigma_{H_{0}}^{2}}} \exp \left[-\frac{1}{2}\left(\gamma-\frac{\beta^{2}}{\alpha}\right)\right]\left[1+\operatorname{erf}\left(\frac{\beta}{\sqrt{2 \alpha}}\right)\right]
$$

$$
{ }^{7} \operatorname{erf}(x)=\frac{2}{\sqrt{\pi}} \int_{0}^{x} e^{-t^{2}} d t
$$



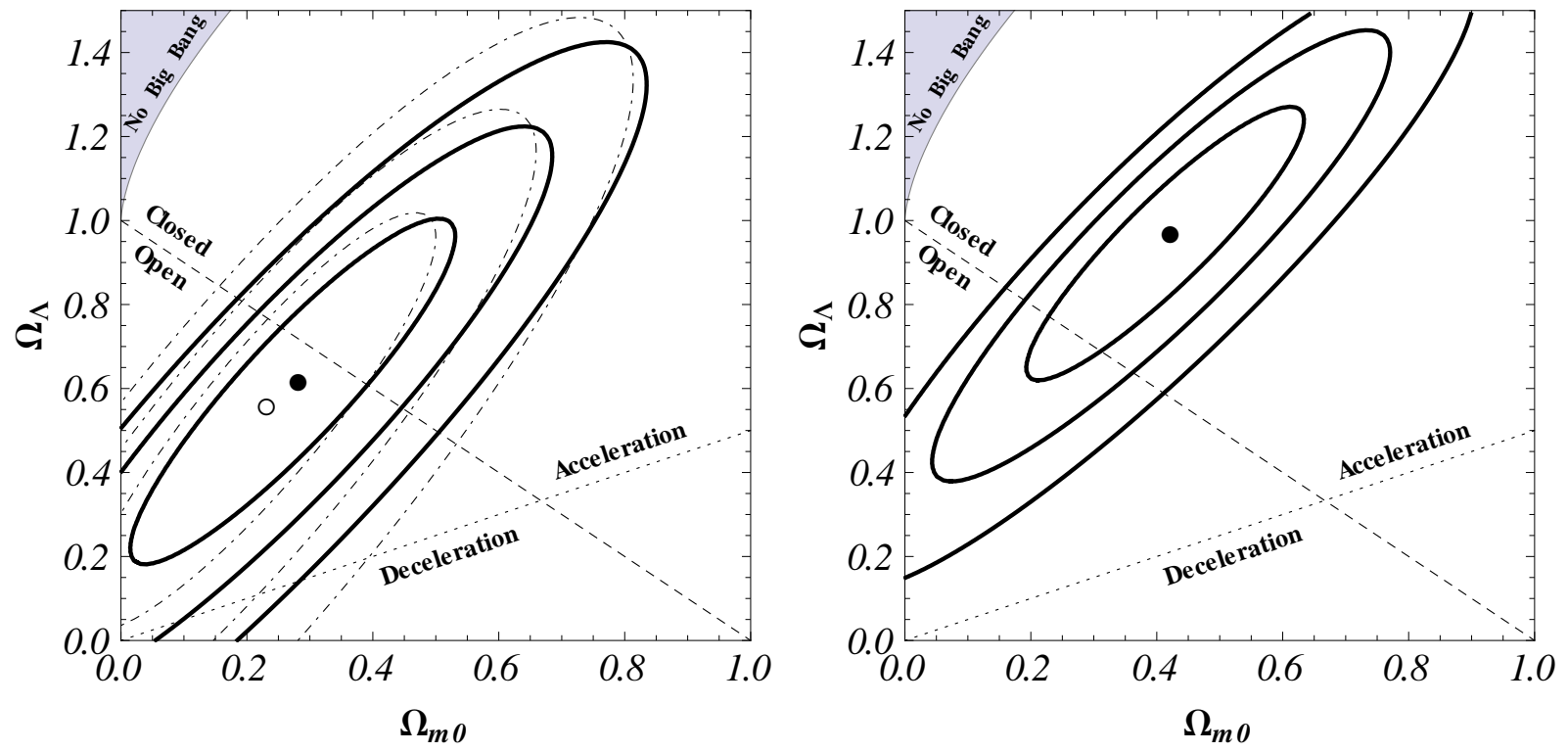

Fig. 1.- Solid lines shows 1,2 , and $3 \sigma$ constraint contours for the $\Lambda$ CDM model from the $H(z)$ data. The left panel is for the $H_{0}=68 \pm 2.8 \mathrm{~km} \mathrm{~s}^{-1} \mathrm{Mpc}^{-1}$ prior and the right panel is for the $H_{0}=73.8 \pm 2.4 \mathrm{~km} \mathrm{~s}^{-1} \mathrm{Mpc}^{-1}$ one. Thin dot-dashed lines in the left panel are 1,2 , and $3 \sigma$ contours reproduced from Chen \& Ratra (2011b), where the prior is $H_{0}=68 \pm 3.5 \mathrm{~km}$ $\mathrm{s}^{-1} \mathrm{Mpc}^{-1}$; the empty circle is the corresponding best-fit point. The dashed diagonal lines correspond to spatially-flat models, the dotted lines demarcate zero-acceleration models, and the shaded area in the upper left-hand corners are the region for which there is no big bang. The filled black circles correspond to best-fit points. For quantitative details see Table 2.

We maximize the likelihood $\mathcal{L}_{H}(\mathbf{p})$, or equivalently minimize $\chi_{H}^{2}(\mathbf{p})=-2 \ln \mathcal{L}_{H}(\mathbf{p})$, with respect to the parameters $\mathbf{p}$ to find the best-fit parameter values $\mathbf{p}_{\mathbf{0}}$. In the models we consider $\chi_{H}^{2}$ depends on two parameters. We define $1 \sigma, 2 \sigma$, and $3 \sigma$ confidence intervals as two-dimensional parameter sets bounded by $\chi_{H}^{2}(\mathbf{p})=\chi_{H}^{2}\left(\mathbf{p}_{\mathbf{0}}\right)+2.3, \chi_{H}^{2}(\mathbf{p})=\chi_{H}^{2}\left(\mathbf{p}_{\mathbf{0}}\right)+6.17$, and $\chi_{H}^{2}(\mathbf{p})=\chi_{H}^{2}\left(\mathbf{p}_{\mathbf{0}}\right)+11.8$, respectively.

Even though the precision of measurements of the Hubble constant have greatly improved over the last decade, the concomitant improvement in the precision of other cosmological measurements means that in some cases the Hubble constant uncertainty still significantly affects cosmological parameter estimation. For a recent example see Calabrese et al. (2012). The values of $\bar{H}_{0} \pm \sigma_{H_{0}}$ that we use in this paper are $68 \pm 2.8 \mathrm{~km} \mathrm{~s}^{-1} \mathrm{Mpc}^{-1}$ and $73.8 \pm 2.4 \mathrm{~km} \mathrm{~s}^{-1} \mathrm{Mpc}^{-1}$. The first is from a median statistics analysis (Gott et al. 2001) of 553 measurements of $H_{0}$ (Chen \& Ratra 2011a); this estimate has been remarkably stable for over a decade now (Gott et al. 2001; Chen et al. 2003). The second value is the most precise recent one, based on HST measurements (Riess et al. 2011). Other recent mea- 

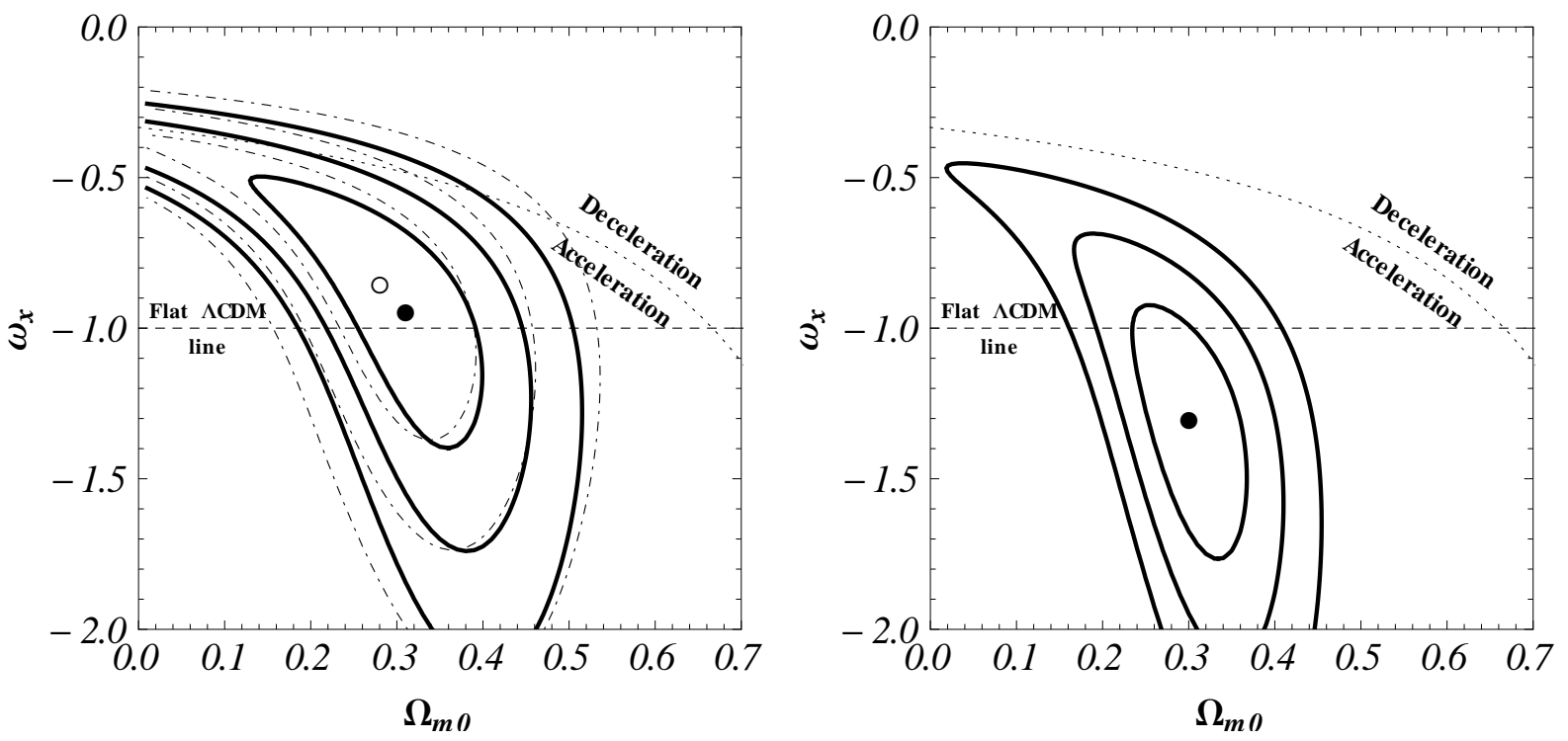

Fig. 2.- Solid lines shows 1, 2, and $3 \sigma$ constraint contours for the XCDM parametrization from the $H(z)$ data. The left panel is for the $H_{0}=68 \pm 2.8 \mathrm{~km} \mathrm{~s}^{-1} \mathrm{Mpc}^{-1}$ prior and the right panel is for the $H_{0}=73.8 \pm 2.4 \mathrm{~km} \mathrm{~s}^{-1} \mathrm{Mpc}^{-1}$ one. Thin dot-dashed lines in the left panel are 1, 2, and $3 \sigma$ contours reproduced from Chen \& Ratra (2011b), where the prior is $H_{0}=68 \pm 3.5 \mathrm{~km} \mathrm{~s}^{-1} \mathrm{Mpc}^{-1}$; the empty circle is the corresponding best-fit point. The dashed horizontal lines at $\omega_{\mathrm{X}}=-1$ correspond to spatially-flat $\Lambda$ CDM models and the curved dotted lines demarcate zero-acceleration models. The filled black circles correspond to best-fit points. For quantitative details see Table 2.

surements are not inconsistent with at least one of the two values we use as a prior (see, e.g., Freedman et al. 2012; Sorce et al. 2012; Tammann \& Reindl 2012).

Figures $1-3$ show the constraints from the $H(z)$ data for the three dark energy models we consider, and for the two different $H_{0}$ priors. Table 2 lists the best fit parameter values. Comparing these plots with Figs. 1-3 of Chen \& Ratra (2011b), whose 1, 2 and $3 \sigma$ constraint contours are reproduced here as dot-dashed lines in the left panels of Figs $1-3$, we see that the contours derived from the new data are more constraining, by about a standard deviation, because of the 8 new, more precise, Moresco et al. (2012a) data points used here. On comparing the left and right panels in these three figures, we see that the constraint contours are quite sensitive to the value of $H_{0}$ used, as well as to the uncertainty associated with the Hubble constant measurement. 

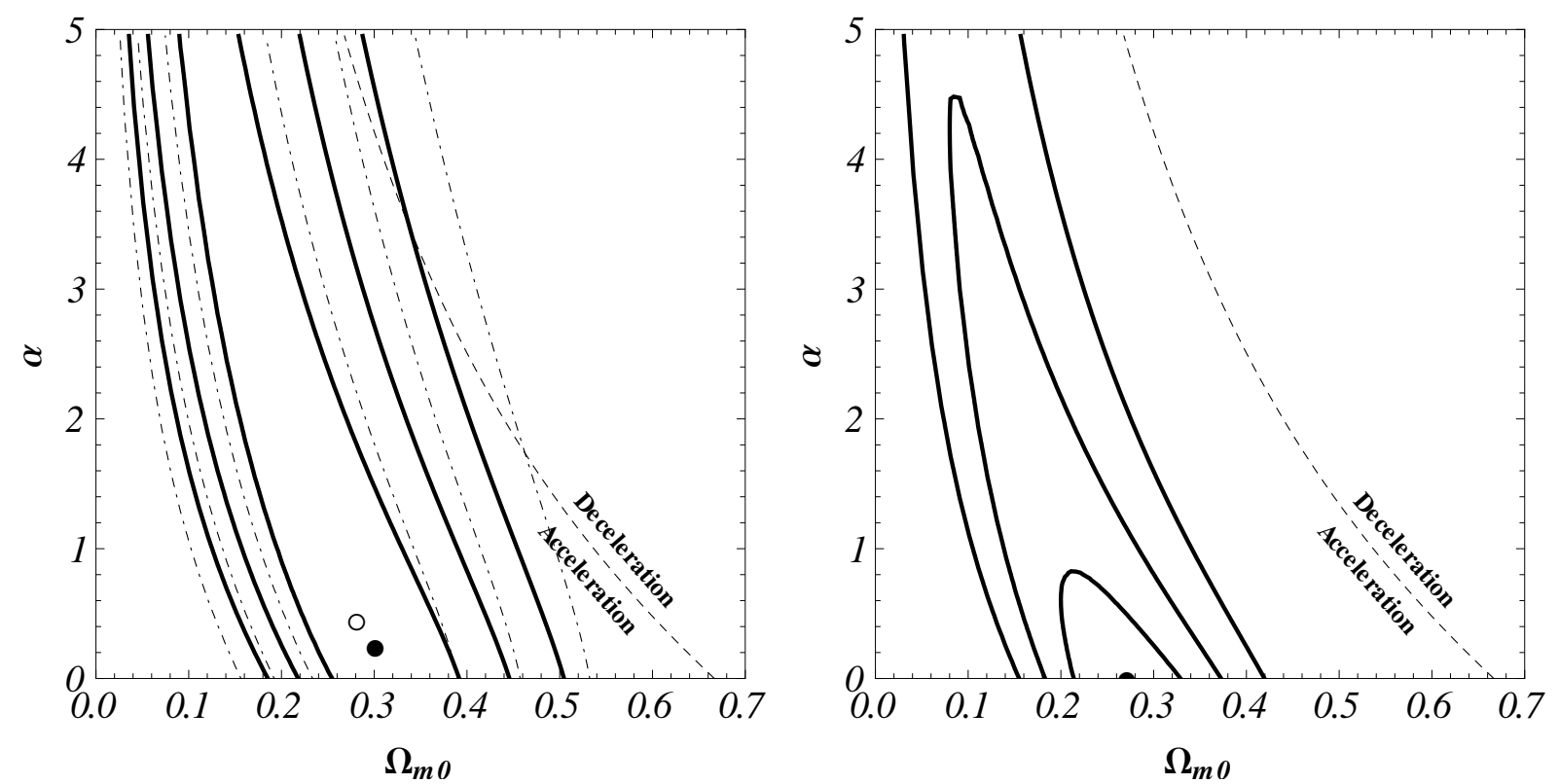

Fig. 3.- Solid lines shows 1, 2, and $3 \sigma$ constraint contours for the $\phi$ CDM model from the $H(z)$ data. The left panel is for the $H_{0}=68 \pm 2.8 \mathrm{~km} \mathrm{~s}^{-1} \mathrm{Mpc}^{-1}$ prior and the right panel is for the $H_{0}=73.8 \pm 2.4 \mathrm{~km} \mathrm{~s}^{-1} \mathrm{Mpc}^{-1}$ one. Thin dot-dashed lines in the left panel are 1, 2, and $3 \sigma$ contours reproduced from Chen \& Ratra (2011b), where the prior is $H_{0}=68 \pm 3.5$ $\mathrm{km} \mathrm{s}^{-1} \mathrm{Mpc}^{-1}$; the empty circle is the corresponding best-fit point. The horizontal axes at $\alpha=0$ correspond to spatially-flat $\Lambda$ CDM models and the curved dotted lines demarcate zeroacceleration models. The filled black circles correspond to best-fit points. For quantitative details see Table 2 .

\section{Constraints from the SNIa data}

While the $H(z)$ data provide tight constraints on a linear combination of cosmological parameters, the very elongated constraint contours of Figs. 1-3 imply that these data alone cannot significantly discriminate between cosmological models. To tighten the constraints we must add other data to the mix.

The second set of data that we use are the Type Ia supernova data from the Suzuki et al. (2012) Union2.1 compilation of 580 SNIa distance modulus $\mu_{\mathrm{obs}}\left(z_{i}\right)$ measurements at measured redshifts $z_{i}$ (covering the redshift range of 0.015 to 1.414 ) with associated one standard deviation uncertainties $\sigma_{i}$. The predicted distance modulus is

$$
\mu_{\mathrm{th}}\left(z_{i} ; H_{0}, \mathbf{p}\right)=\underbrace{5 \log _{10}(3000 y(z)(1+z))+25}_{=\mu_{0}}-5 \log _{10}(h),
$$




\begin{tabular}{|c|c|c|c|c|c|c|}
\hline \multirow[b]{2}{*}{ Model and prior } & \multicolumn{2}{|r|}{$H(z)$} & \multicolumn{2}{|r|}{ SNeIa } & \multicolumn{2}{|r|}{$\mathrm{BAO}$} \\
\hline & $\chi_{\min }^{2}$ & B.F.P & & B.F.P & $\chi_{\min }^{2}$ & B.F.P \\
\hline $\begin{array}{c}\Lambda \mathrm{CDM} \\
h=0.68 \pm 0.028\end{array}$ & 14.6 & $\begin{array}{c}\Omega_{m 0}=0.28 \\
\Omega_{\Lambda}=0.62\end{array}$ & & $\Omega_{m 0}=0.29$ & & $\Omega_{m 0}=0.27$ \\
\hline $\begin{array}{c}\Lambda \mathrm{CDM} \\
h=0.738 \pm 0.024\end{array}$ & 14.6 & $\begin{array}{c}\Omega_{m 0}=0.42 \\
\Omega_{\Lambda}=0.97\end{array}$ & & $\Omega_{\Lambda}=0.69$ & 5.5 & $\Omega_{\Lambda}=0.87$ \\
\hline $\begin{array}{c}\mathrm{XCDM} \\
h=0.68 \pm 0.028\end{array}$ & 14.6 & $\begin{array}{c}\Omega_{m 0}=0.31 \\
\omega_{X}=-0.94\end{array}$ & & $\Omega_{m 0}=0.29$ & & $\Omega_{m 0}=0.27$ \\
\hline $\begin{array}{c}\text { XCDM } \\
h=0.738 \pm 0.024\end{array}$ & 14.6 & $\begin{array}{l}\Omega_{m 0}=0.30 \\
\omega_{X}=-1.3\end{array}$ & & $\omega_{X}=-0.99$ & & $\omega_{X}=-1.21$ \\
\hline $\begin{array}{c}\phi \mathrm{CDM} \\
h=0.68 \pm 0.028\end{array}$ & 14.6 & $\begin{array}{c}\Omega_{m 0}=0.30 \\
\alpha=0.25\end{array}$ & & $\Omega_{m 0}=0.27$ & & $\Omega_{m 0}=0.30$ \\
\hline $\begin{array}{c}\phi \mathrm{CDM} \\
h=0.738 \pm 0.024\end{array}$ & 15.6 & $\begin{array}{c}\Omega_{m 0}=0.27 \\
\alpha=0.00\end{array}$ & 045 & $\alpha=0.20$ & 0.9 & $\alpha=0.00$ \\
\hline
\end{tabular}

Table 2: The minimum value of $\chi^{2}$ and the corresponding best-fit points (B.F.P) which maximize the likelihood for the three individual data sets. The SNIa values are for the case including systematic errors. Ignoring SNIa systematic errors, for the $\Lambda$ CDM model $\chi_{S N}^{2}\left(\mathbf{p}_{\mathbf{0}}\right)=562$, at $\left(\Omega_{m 0}, \Omega_{\Lambda}\right)=(0.28,0.73)$; for the XCDM case $\chi_{S N}^{2}\left(\mathbf{p}_{\mathbf{0}}\right)=562$ at $\left(\Omega_{m 0}, \omega_{\mathrm{X}}\right)=(0.28,-1.01)$; and for the $\phi \mathrm{CDM}$ model $\chi_{S N}^{2}\left(\mathbf{p}_{\mathbf{0}}\right)=562$, at $\left(\Omega_{m 0}, \alpha\right)=(0.27,0.05)$.

where $H_{0}=100 h \mathrm{~km} \mathrm{~s}^{-1} \mathrm{Mpc}^{-1}$ and $y(z)$ is the dimensionless coordinate distance,

$$
y(z)= \begin{cases}\frac{a_{0} H_{0}}{K} \sin \left(\frac{K}{a_{0} H_{0}} \int_{0}^{z} \frac{d z^{\prime}}{E\left(z^{\prime}\right)}\right) & K^{2}>0 \\ \int_{0}^{z} \frac{d z^{\prime}}{E\left(z^{\prime}\right)} & K^{2}=0 \\ \frac{a_{0} H_{0}}{\sqrt{-K^{2}}} \sinh \left(\frac{\sqrt{-K^{2}}}{a_{0} H_{0}} \int_{0}^{z} \frac{d z^{\prime}}{E\left(z^{\prime}\right)}\right) & K^{2}<0 .\end{cases}
$$

As the SNIa distance modulus measurements $\mu_{\mathrm{obs}}$ are correlated, $\chi^{2}$ is defined as

$$
\chi_{S N}^{2}(h, \mathbf{p})=\Delta \boldsymbol{\mu}^{T} \mathcal{C}^{-1} \Delta \boldsymbol{\mu} .
$$

Here $\Delta \boldsymbol{\mu}$ is a vector of differences $\Delta \mu_{i}=\mu_{\mathrm{th}}\left(z_{i} ; H_{0}, \mathbf{p}\right)-\mu_{\mathrm{obs}}\left(z_{i}\right)$, and $\mathcal{C}^{-1}$ is the inverse of the 580 by 580 Union 2.1 compilation covariance matrix. In index notation,

$$
\chi_{S N}^{2}(h, \mathbf{p})=\sum_{\alpha, \beta}\left[\mu_{0}-5 \log _{10} h-\mu_{\mathrm{obs}}\right]_{\alpha}\left(\mathcal{C}^{-1}\right)_{\alpha \beta}\left[\mu_{0}-5 \log _{10} h-\mu_{\mathrm{obs}}\right]_{\beta} .
$$


The covariance matrix is symmetric so this can be written as

$$
\chi_{S N}^{2}(h, \mathbf{p})=A(\mathbf{p})-10 B(\mathbf{p}) \log _{10}(h)+25 C\left[\log _{10}(h)\right]^{2}
$$

where

$$
\begin{aligned}
& A(\mathbf{p})=\sum_{\alpha, \beta}\left(\mu_{0}-\mu_{\mathrm{obs}}\right)_{\alpha}\left(\mathcal{C}^{-1}\right)_{\alpha \beta}\left(\mu_{0}-\mu_{\mathrm{obs}}\right)_{\beta} \\
& B(\mathbf{p})=\sum_{\alpha}\left(\mu_{0}-\mu_{\mathrm{obs}}\right)_{\alpha} \sum_{\beta}\left(\mathcal{C}^{-1}\right)_{\alpha \beta} \\
& C=\sum_{\alpha, \beta}\left(\mathcal{C}^{-1}\right)_{\alpha \beta} .
\end{aligned}
$$

The corresponding likelihood function, when considering a flat $H_{0}$ prior, is

$$
\mathcal{L}_{S N}(\mathbf{p})=\int_{0}^{\infty} e^{-\chi_{S N}^{2}(h, \mathbf{p}) / 2} d h
$$

Defining

$$
\delta=\frac{25 C}{2(\ln 10)^{2}}, \quad \varepsilon=\frac{B(\mathbf{p}) \ln 10}{5 C},
$$

the above integral takes the form

$$
\mathcal{L}_{S N}(\mathbf{p})=\sqrt{\frac{\pi}{\delta}} \exp \left[-\frac{1}{2}\left(A(\mathbf{p})-\frac{B^{2}(\mathbf{p})}{C}-2 \varepsilon-\frac{1}{2 \delta^{2}}\right)\right] .
$$

The $h$-independent

$$
\chi_{S N}^{2}(\mathbf{p})=-2 \ln \mathcal{L}_{S N}(\mathbf{p})=A(\mathbf{p})-\frac{B^{2}(\mathbf{p})}{C}-\frac{2 \ln (10)}{5 C} B(\mathbf{p})-Q
$$

where $Q$ is a constant that does not depend on the model parameters $\mathbf{p}$,

$$
Q=\frac{2(\ln 10)^{4}}{625 C^{2}}+2 \ln \left(\frac{2 \pi(\ln 10)^{2}}{25 C}\right)
$$

and so can be ignored. We minimize $\chi_{S N}^{2}(\mathbf{p})$ with respect to the model parameters $\mathbf{p}$ to find the best-fit parameter values $\mathbf{p}_{\mathbf{0}}$ and constraint contours.

Figure 4 shows constraints from the SNIa data on the three dark energy models we consider here. For the $\Lambda$ CDM model and the XCDM parametrization the constraints shown in Fig. 4 are in very good agreement with those in Figs. 5 and 6 of Suzuki et al. (2012). The $\phi \mathrm{CDM}$ model SNIa data constraints shown in Fig. 4 have not previously been computed. Comparing the SNIa constraints of Fig. 4 to those which follow from the $H(z)$ data, Figs. $1-3$, it is clear that SNIa data provide tighter constraints on the $\Lambda$ CDM model. For the 

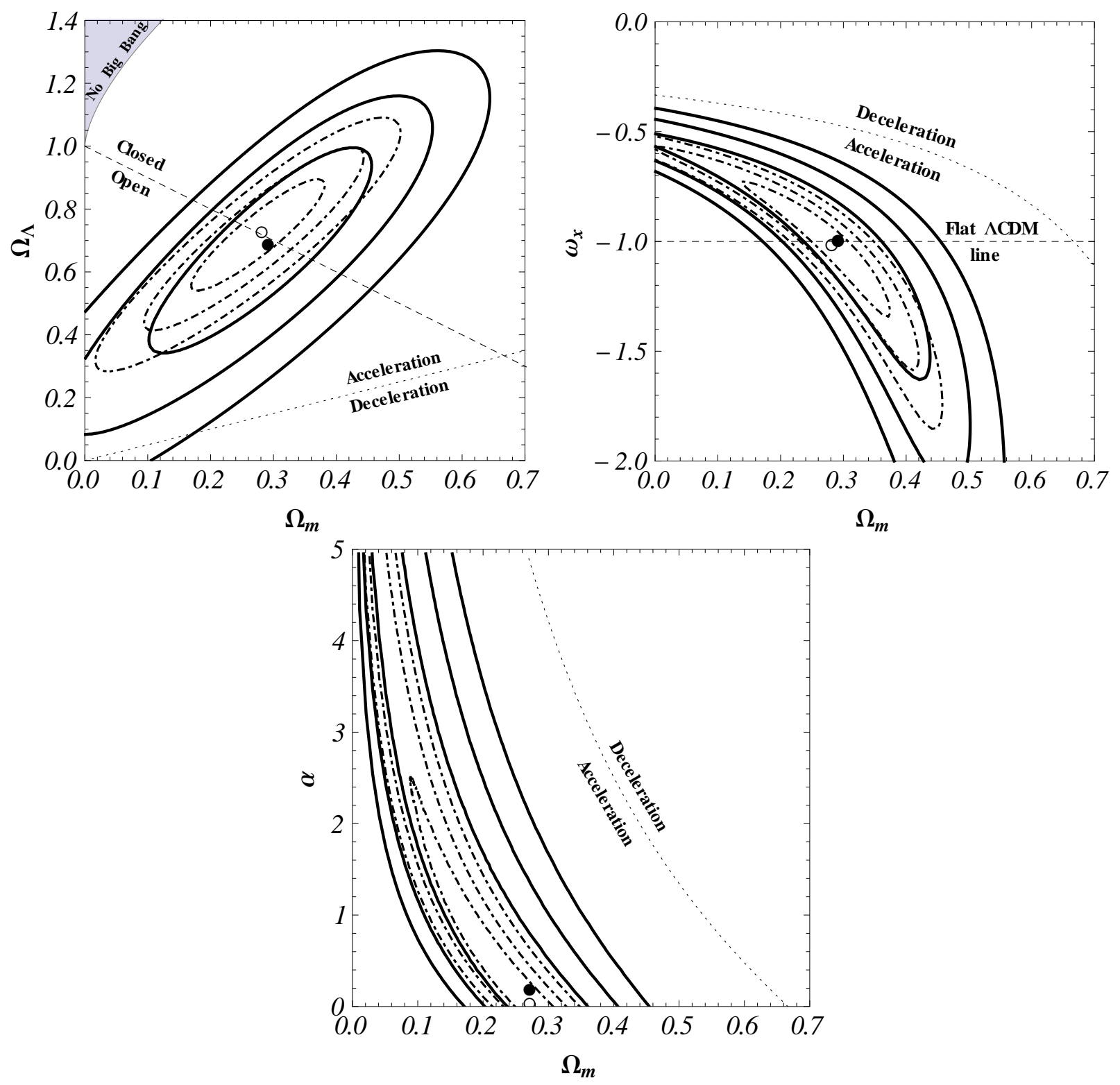

Fig. 4. - Thick solid (dot-dashed) lines are 1, 2, and 3- $\sigma$ constraint contours from SNIa data with (without) systematic errors. Filled (open) circles demarcate likelihood maxima for the case of data with (without) systematic errors. The top left plot is for the $\Lambda$ CDM model, the top right plot is for the XCDM parametrization, and the bottom one is for the $\phi \mathrm{CDM}$ model. For quantitative details see Table 2. 
XCDM case both SNIa data and $H(z)$ data provide approximately similar constraints, while the SNIa constraints are somewhat more restrictive than the $H(z)$ ones for the $\phi$ CDM model. However, in general, the SNIa constraints are not very significantly more restrictive than the $H(z)$ constraints, which is a remarkable result. It is also reassuring that both data favor approximately similar regions of parameters space, for all three models we consider. However, given that the degeneracy in parameter space is similar for the $H(z)$ and SNIa data, a joint analysis of just these two data sets is unlikely to greatly improve the constraints.

\section{Constraints from the BAO data}

In an attempt to further tighten the cosmological parameter constraints, we now include BAO data in the analysis. To constrain cosmological parameters using BAO data we follow the procedure of Blake et al. (2011). To derive the BAO constraints we make use of the distance parameter $D_{V}(z)$, a combination of the angular diameter distance and the Hubble parameter, given by

$$
D_{V}(z)=\left[(1+z)^{2} d_{A}(z)^{2} \frac{c z}{H(z)}\right]^{1 / 3} .
$$

Here $d_{A}(z)$ is the angular diameter distance

$$
d_{A}(z)=\frac{y(z)}{H_{0}(1+z)}
$$

where $y(z)$ is the dimensionless coordinate distance given in Eq. (20).

We use measurements of the acoustic parameter $A(z)$ from Blake et al. (2011), where the theoretically-predicted $A_{\mathrm{th}}(z)$ is given in Eq. (5) of Eisenstein et al. (2005),

$$
A_{\mathrm{th}}(z)=\frac{100 D_{V}(z) \sqrt{\Omega_{m} h^{2}}}{z} .
$$

Using Eqs. (28)-(30) we have

$$
A_{\mathrm{th}}(z)=\sqrt{\Omega_{m}}\left[\frac{y^{2}(z)}{z^{2} E(z)}\right]^{1 / 3},
$$

which is $h$ independent and where $E(z)$ is defined in Sec. 2.

Using the WiggleZ $A_{\mathrm{obs}}(z)$ data from Table 3 of Blake et al. (2011), we compute

$$
\chi_{A_{z}}^{2}(\mathbf{p})=\Delta \mathbf{A}^{T}\left(\mathrm{C}_{A_{z}}\right)^{-1} \Delta \mathbf{A} .
$$



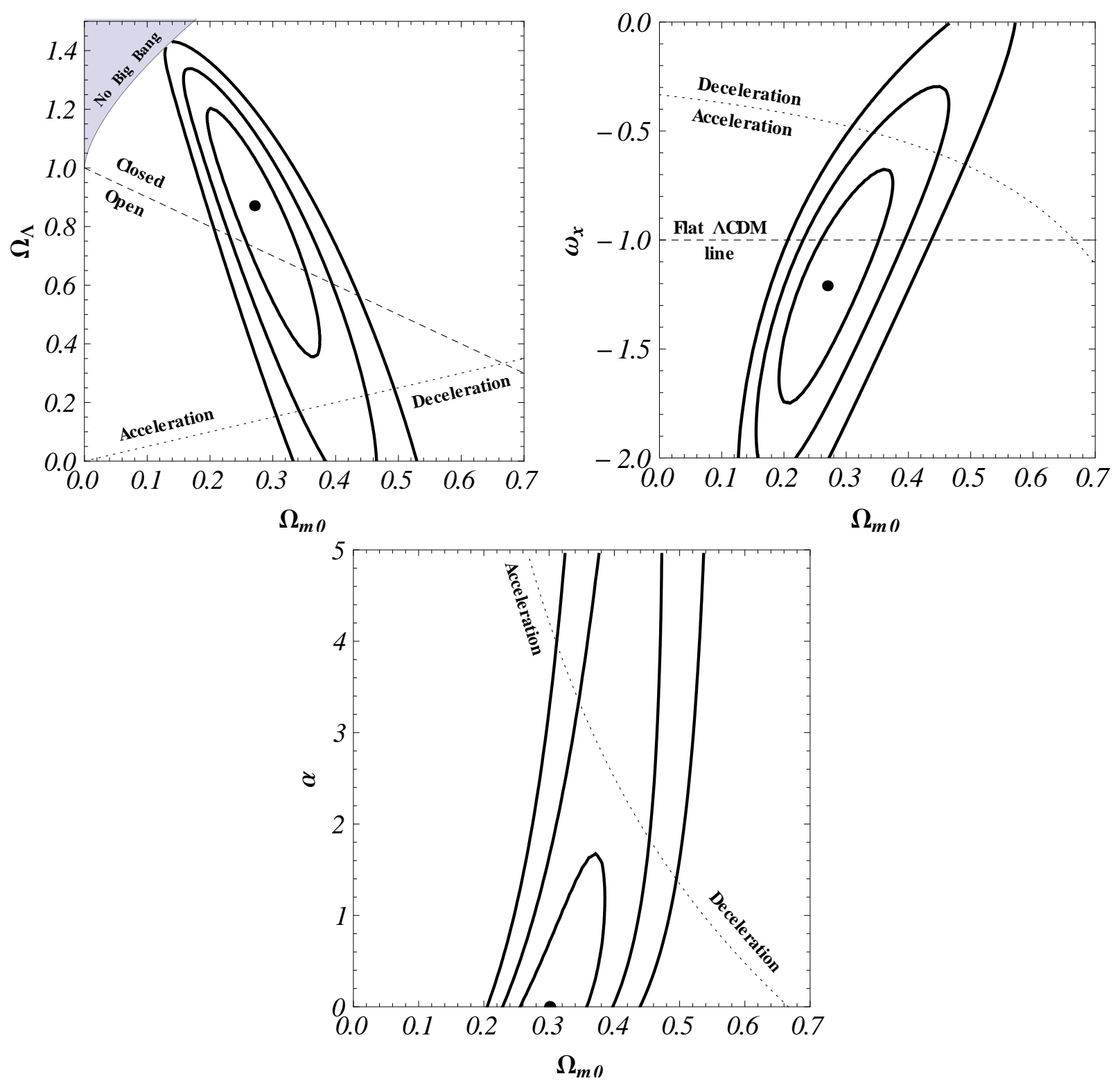

Fig. 5.- 1, 2, and $3 \sigma$ constraint contours from the BAO data. Filled circles denote likelihood maxima. The top left plot is for the $\Lambda \mathrm{CDM}$ model, the top right one is for the $\mathrm{XCDM}$ parametrization, and the bottom plot is for the $\phi \mathrm{CDM}$ model. For quantitative details see Table 2 . 
Here $\Delta \mathbf{A}$ is a vector consisting of differences $\Delta A_{i}=A_{\mathrm{th}}\left(z_{i} ; \mathbf{p}\right)-A_{\mathrm{obs}}\left(z_{i}\right)$ and $\left(\mathrm{C}_{A z}\right)^{-1}$ is the inverse of the 3 by 3 covariance matrix given in Table 3 of Blake et al. (2011).

We also use the 6dFGS and SDSS data, three measurements from Beutler et al. (2011) and Percival et al. (2010), listed in Blake et al. (2011). In this case the distilled parameter

$$
d_{\mathrm{th}}(z)=\frac{r_{s}\left(z_{d}\right)}{D_{V}(z)}
$$

where $r_{s}\left(z_{d}\right)$ is the sound horizon at the drag epoch, is given in Eq. (6) of Eisenstein \& Hu (1998). The correlation coefficients for this case are also given in Table 3 of Blake et al. (2011). Using the covariance matrix we define

$$
\chi_{d_{z}}^{2}(h, \mathbf{p})=\Delta \mathbf{d}^{T}\left(\mathrm{C}_{d_{z}}\right)^{-1} \Delta \mathbf{d}
$$

where $\Delta \mathbf{d}$ is a vector consisting of differences $\Delta d_{i}=d_{\mathrm{th}}\left(z_{i} ; h, \mathbf{p}\right)-d_{\mathrm{obs}}\left(z_{i}\right)$ and $\mathrm{C}_{d_{z}}$ is the the covariance matrix (Blake et al. 2011). We then marginalize over a flat prior for $H_{0}$ to get

$$
\chi_{d_{z}}^{2}(\mathbf{p})=-2 \ln \left[\int_{0}^{\infty} e^{-\chi_{d_{z}}^{2}(h, \mathbf{p}) / 2} d h\right]
$$

Since $\chi_{A_{z}}^{2}(\mathbf{p})$ and $\chi_{d_{z}}^{2}(\mathbf{p})$ correspond to independent data, the combined BAO data

$$
\chi_{B A O}^{2}(\mathbf{p})=\chi_{A_{z}}^{2}(\mathbf{p})+\chi_{d_{z}}^{2}(\mathbf{p}) .
$$

We can maximize the likelihood by minimizing $\chi_{B A O}^{2}(\mathbf{p})$ with respect to the model parameters $\mathbf{p}$ to get best-fit parameter values $\mathbf{p}_{\mathbf{0}}$ and constraint contours. Figure 5 show the constraints from the BAO data on the three dark energy models we consider here. The XCDM parametrization constraints shown in this figure are in good agreement with those shown in Fig. 13 of Blake et al. (2011). The constraints shown in the other two panels of Fig. 5 have not previously been computed. Comparing to the $H(z)$ and SNIa constraint contours of Figs. 1-4, we see that the BAO contours are also very elongated, although largely orthogonal to the $H(z)$ and SNIa ones. Consequently, a joint analysis of these data will result in significantly tighter constraints than those derived using any one of these data sets.

\section{Joint constraints}

To constrain cosmological parameters from a joint analysis of the $H(z)$, SNIa, and BAO data we compute

$$
\chi^{2}(\mathbf{p})=\chi_{H}^{2}(\mathbf{p})+\chi_{S N}^{2}(\mathbf{p})+\chi_{B A O}^{2}(\mathbf{p})
$$



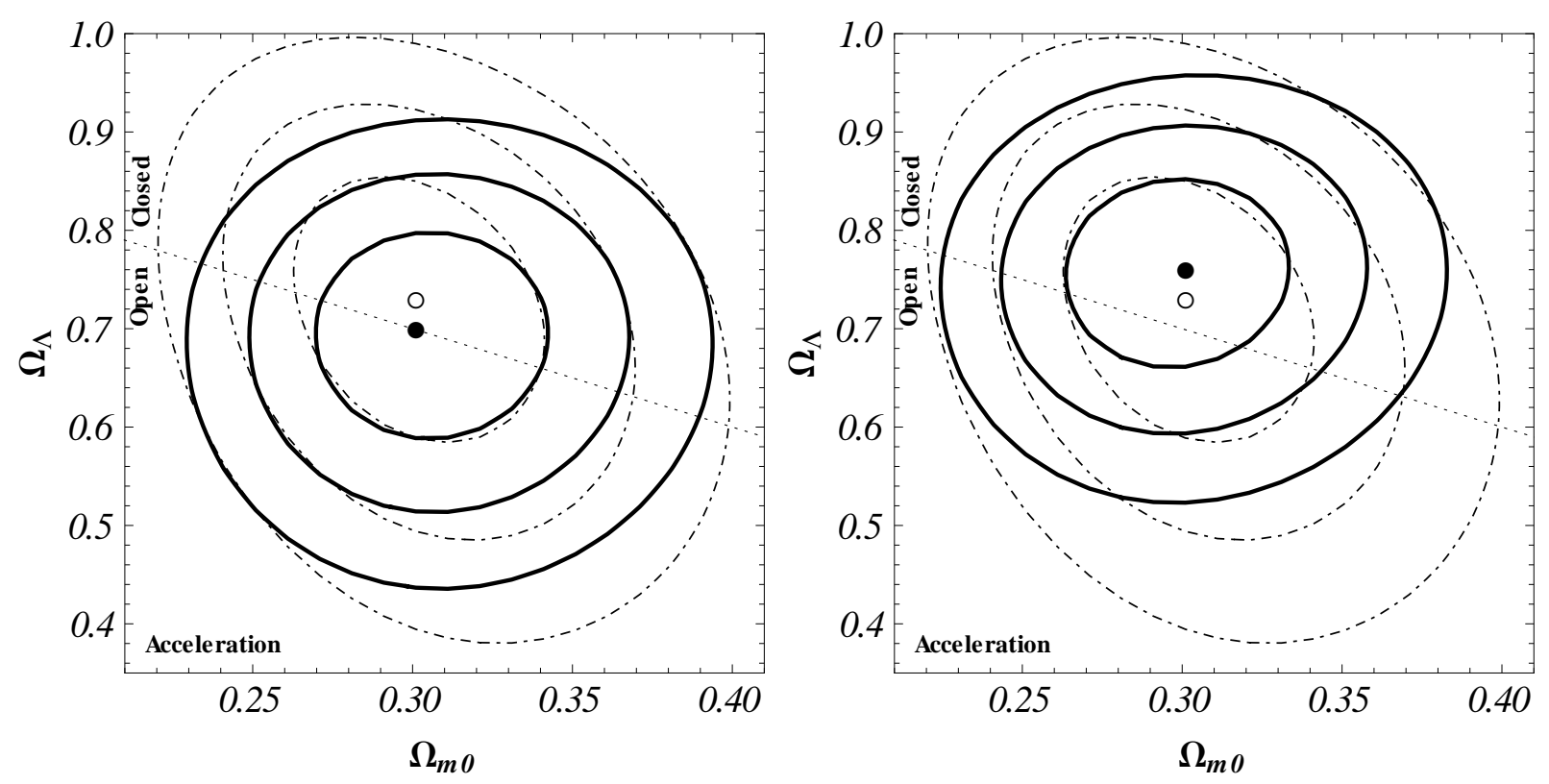

Fig. 6. - Thick solid (dot-dashed) lines are 1, 2, and $3 \sigma$ constraint contours for the $\Lambda$ CDM model from a joint analysis of the BAO and SNIa (with systematic errors) data, with (without) the $H(z)$ data. The full (empty) circle marks the best-fit point determined from the joint analysis with (without) the $H(z)$ data. The dotted sloping line corresponds to spatiallyflat $\Lambda$ CDM models. In the left panel we use the $H_{0}=68 \pm 2.8 \mathrm{~km} \mathrm{~s}^{-1} \mathrm{Mpc}^{-1}$ prior while the right panel is for the $H_{0}=73.8 \pm 2.4 \mathrm{~km} \mathrm{~s}^{-1} \mathrm{Mpc}^{-1}$ case. For quantitative details see Table 3.

for each of the three cosmological models considered here. We minimize $\chi^{2}(\mathbf{p})$ with respect to model parameters $\mathbf{p}$ to get best-fit parameter values $\mathbf{p}_{\mathbf{0}}$ and constraint contours.

Figures $6-8$ show constraints on the cosmological parameters for the $\Lambda \mathrm{CDM}$ and $\phi \mathrm{CDM}$ models and the XCDM parametrization, from a joint analysis of the BAO and SNIa data, as well as from a joint analysis of the BAO, SNIa and $H(z)$ data. Table 3 lists information about best-fit parameter values. Including the $H(z)$ data in the analysis tightens the constraints by more than one standard deviation, in parts of the parameter spaces.

Adding the $H(z)$ data for the $\bar{H}_{0} \pm \sigma_{H_{0}}=68 \pm 2.8 \mathrm{~km} \mathrm{~s}^{-1} \mathrm{Mpc}^{-1}$ prior case improved the constraints most significantly in the $\Lambda$ CDM case (by more than $1 \sigma$ on $\Omega_{\Lambda}$ in parts of parameter space), Fig. 6, and least significantly for the $\phi \mathrm{CDM}$ model, Fig. 8. For the case of the $\bar{H}_{0} \pm \sigma_{H_{0}}=73.8 \pm 2.4 \mathrm{~km} \mathrm{~s}^{-1} \mathrm{Mpc}^{-1}$ prior, adding $H(z)$ again tightens up the constraints the most for the $\Lambda$ CDM model (by more than $1 \sigma$ on $\Omega_{\Lambda}$ ), Fig. 6 , and least so for the XCDM parametrization, Fig. 7. 

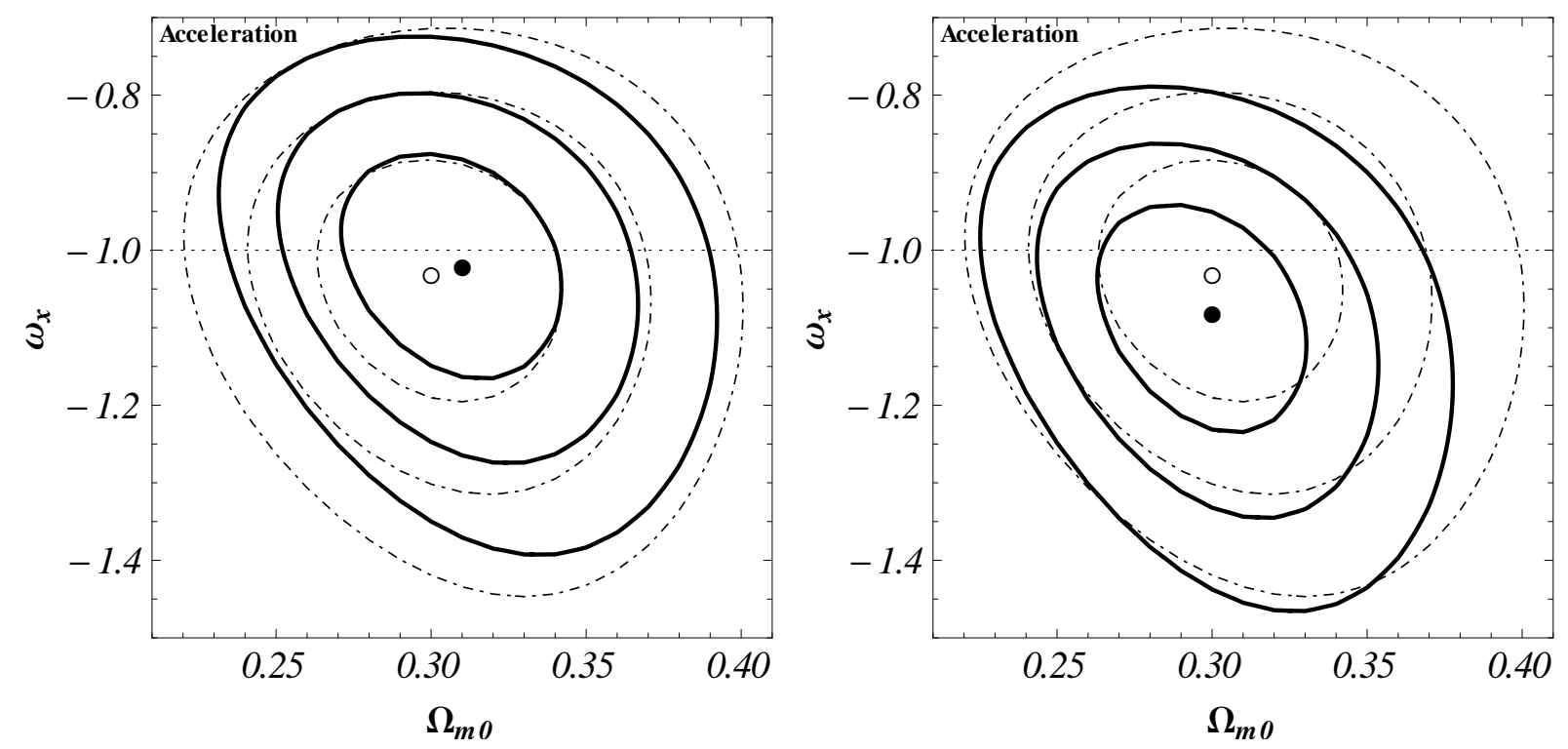

Fig. 7.- Thick solid (dot-dashed) lines are 1, 2, and $3 \sigma$ constraint contours for the XCDM parametrization from a joint analysis of the BAO and SNIa (with systematic errors) data, with (without) the $H(z)$ data. The full (empty) circle marks the best-fit point determined from the joint analysis with (without) the $H(z)$ data. The dotted horizontal line at $\omega_{\mathrm{X}}=-1$ corresponds to spatially-flat $\Lambda$ CDM models. In the left panel we use the $H_{0}=68 \pm 2.8 \mathrm{~km}$ $\mathrm{s}^{-1} \mathrm{Mpc}^{-1}$ prior while the right panel is for the $H_{0}=73.8 \pm 2.4 \mathrm{~km} \mathrm{~s}^{-1} \mathrm{Mpc}^{-1}$ case. For quantitative details see Table 3 .

Figures 9-11 show the constraints on the cosmological parameters of the three models, from a joint analysis of the $\mathrm{BAO}$ and $H(z)$ data, as well as from a joint analysis of the three data sets. Table 3 lists the best-fit parameter values. Comparing these figures to Figs. $6-8$ allows for a comparison between the discriminating power of the SNIa and $H(z)$ data.

Figure 9 shows that adding SNIa data to the $H(z)$ and BAO data combination for the $\bar{H}_{0} \pm \sigma_{H_{0}}=68 \pm 2.8 \mathrm{~km} \mathrm{~s}^{-1} \mathrm{Mpc}^{-1}$ prior case tightens up the constraints by more than $1 \sigma$ on $\Omega_{\Lambda}$ from below, while addition of SNIa data for the $\bar{H}_{0} \pm \sigma_{H_{0}}=73.8 \pm 2.4 \mathrm{~km} \mathrm{~s}^{-1} \mathrm{Mpc}^{-1}$ prior case tightens up the constraints by more than $1 \sigma$ on $\Omega_{\Lambda}$ from above. Addition of SNIa data to the $H(z)$ and BAO combination doesn't much improve the constraints on $\Omega_{m 0}$ for either prior.

Figures 9-11 show that adding SNIa data to the $H(z)$ and BAO combination results in the most prominent effect for the XCDM case, Fig. 10. Here for the $\bar{H}_{0} \pm \sigma_{H_{0}}=68 \pm 2.8 \mathrm{~km}$ $\mathrm{s}^{-1} \mathrm{Mpc}^{-1}$ prior it tightens up the constraints by more than $1 \sigma$ on $\omega_{X}$ from above and below while for the $\bar{H}_{0} \pm \sigma_{H_{0}}=73.8 \pm 2.4 \mathrm{~km} \mathrm{~s}^{-1} \mathrm{Mpc}^{-1}$ prior it tightens up the constraints by 

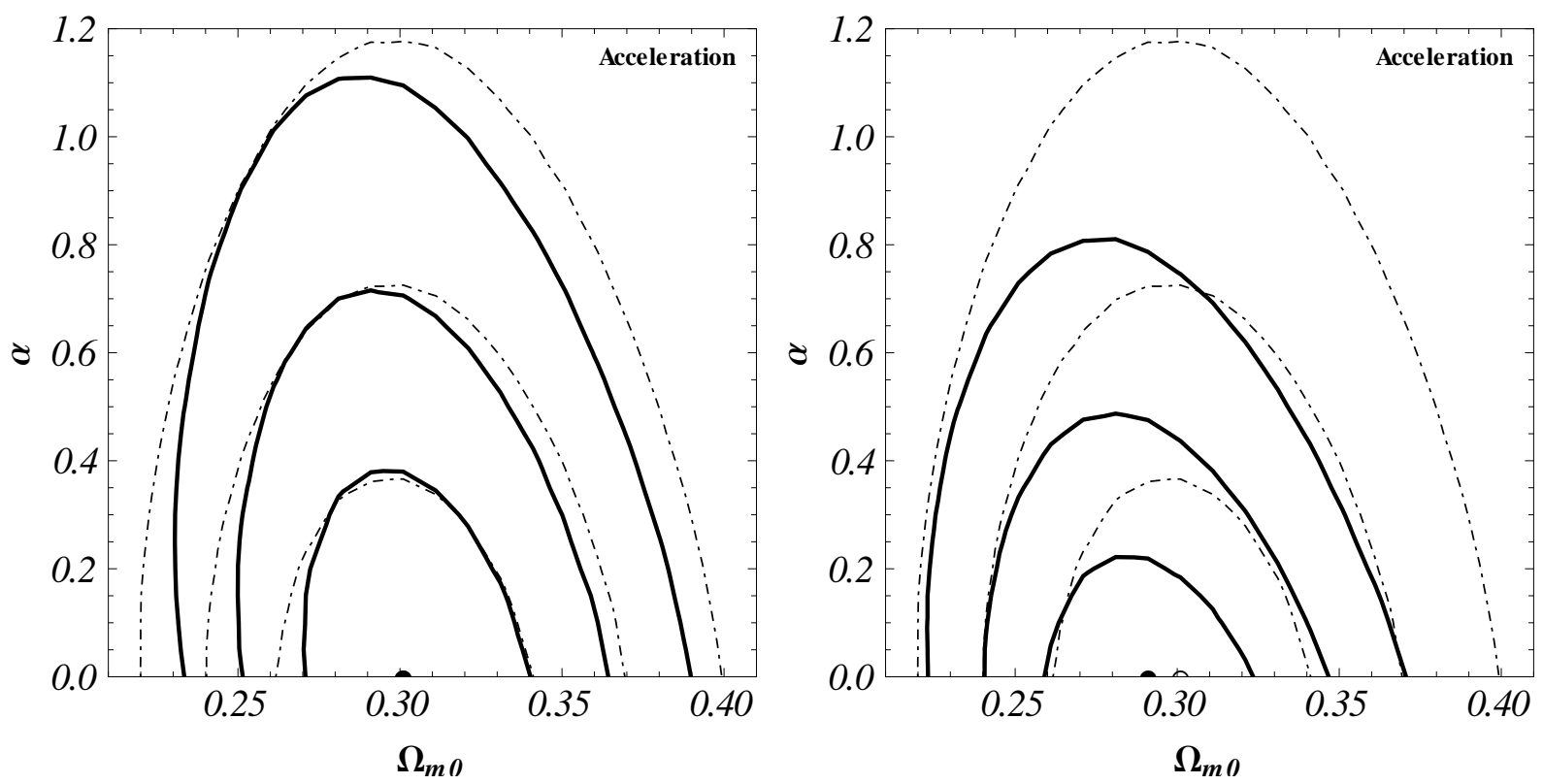

Fig. 8. - Thick solid (dot-dashed) lines are 1, 2, and $3 \sigma$ constraint contours for the $\phi \mathrm{CDM}$ model from a joint analysis of the BAO and SNIa (with systematic errors) data, with (without) the $H(z)$ data. The full (empty) circle marks the best-fit point determined from the joint analysis with (without) the $H(z)$ data (in the left panel the full and empty circles overlap). The $\alpha=0$ horizontal axes correspond to spatially-flat $\Lambda$ CDM models. In the left panel we use the $H_{0}=68 \pm 2.8 \mathrm{~km} \mathrm{~s}^{-1} \mathrm{Mpc}^{-1}$ prior while the right panel is for the $H_{0}=$ $73.8 \pm 2.4 \mathrm{~km} \mathrm{~s}^{-1} \mathrm{Mpc}^{-1}$ case. For quantitative details see Table 3 .

more than $2 \sigma$ on $\omega_{X}$ from below. Addition of SNIa data to the $H(z)$ and BAO combination doesn't much improve the constraints on $\Omega_{m 0}$ for either prior in this case.

In the $\phi \mathrm{CDM}$ case, Fig. 11, adding SNIa data to $H(z)$ and BAO combination affects the constraint on $\alpha$ the most for the $\bar{H}_{0} \pm \sigma_{H_{0}}=68 \pm 2.8 \mathrm{~km} \mathrm{~s}^{-1} \mathrm{Mpc}^{-1}$ prior case. The effect on $\Omega_{m 0}$ is little stronger than what happens in the $\Lambda$ CDM and XCDM cases but still less than $1 \sigma$.

Table 4 lists the two standard deviation bounds on the individual cosmological parameters, determined from their one-dimensional posterior probability distributions functions (which are derived by marginalizing the two-dimensional likelihood over the other cosmological parameter) for different combinations of data set.

The constraints on the cosmological parameters that we derive from only the BAO and SNIa data are restrictive, but less so than those shown in Fig. 4 of Chen \& Ratra (2011b). This is because the new Suzuki et al. (2012) SNIa compilation data we use here is based 


\begin{tabular}{|c|c|c|c|c|c|c|}
\hline \multirow[b]{2}{*}{ Model and prior } & \multicolumn{2}{|c|}{$H(z)+\mathrm{BAO}$} & \multicolumn{2}{|c|}{$H(z)+\mathrm{SNIa}+\mathrm{BAO}$} & \multicolumn{2}{|c|}{$\mathrm{SNIa}+\mathrm{BAO}$} \\
\hline & $\chi_{\min }^{2}$ & B.F.P & $\chi_{\min }^{2}$ & B.F.P & $\chi_{\min }^{2}$ & B.F.P \\
\hline $\begin{array}{c}\Lambda \mathrm{CDM} \\
h=0.68 \pm 0.028\end{array}$ & 20.7 & $\begin{array}{c}\Omega_{m 0}=0.31 \\
\Omega_{\Lambda}=0.68\end{array}$ & 566 & $\begin{array}{c}\Omega_{m 0}=0.30 \\
\Omega_{\Lambda}=0.70\end{array}$ & \multirow{2}{*}{551} & $\Omega_{m 0}=0.30$ \\
\hline $\begin{array}{c}\Lambda \mathrm{CDM} \\
h=0.738 \pm 0.024\end{array}$ & 21.0 & $\begin{array}{c}\Omega_{m 0}=0.29 \\
\Omega_{\Lambda}=0.79\end{array}$ & 567 & $\begin{array}{c}\Omega_{m 0}=0.30 \\
\Omega_{\Lambda}=0.76\end{array}$ & & $\Omega_{\Lambda}=0.73$ \\
\hline $\begin{array}{c}\text { XCDM } \\
h=0.68 \pm 0.028\end{array}$ & 20.7 & $\begin{array}{l}\Omega_{m 0}=0.31 \\
\omega_{X}=-0.99\end{array}$ & 566 & $\begin{array}{l}\Omega_{m 0}=0.31 \\
\omega_{X}=-1.02\end{array}$ & \multirow{2}{*}{551} & $\Omega_{m 0}=0.30$ \\
\hline $\begin{array}{c}\text { XCDM } \\
h=0.738 \pm 0.024\end{array}$ & 20.8 & $\begin{array}{l}\Omega_{m 0}=0.28 \\
\omega_{X}=-1.19\end{array}$ & 567 & $\begin{array}{l}\Omega_{m 0}=0.30 \\
\omega_{X}=-1.08\end{array}$ & & $\omega_{X}=-1.03$ \\
\hline $\begin{array}{c}\phi \mathrm{CDM} \\
h=0.68 \pm 0.028\end{array}$ & 20.7 & $\begin{array}{c}\Omega_{m 0}=0.31 \\
\alpha=0.05\end{array}$ & 566 & $\begin{array}{c}\Omega_{m 0}=0.30 \\
\alpha=0.00\end{array}$ & \multirow{2}{*}{551} & $\Omega_{m 0}=0.30$ \\
\hline $\begin{array}{c}\phi \mathrm{CDM} \\
h=0.738 \pm 0.024\end{array}$ & 22.0 & $\begin{array}{c}\Omega_{m 0}=0.29 \\
\alpha=0.00\end{array}$ & 567 & $\begin{array}{c}\Omega_{m 0}=0.29 \\
\alpha=0.00\end{array}$ & & $\alpha=0.00$ \\
\hline
\end{tabular}

Table 3: The minimum value of $\chi^{2}$ and the corresponding best fit points (B.F.P) which maximize the likelihood, for different combinations of data. The SNIa data values are for the case including systematic errors.

on a more careful accounting of the systematic errors, which have increased. Consequently, including the $H(z)$ data, in addition to the BAO and SNIa data, in the analysis, more significantly tightens the constraints: compare Figs. $6-8$ here to Figs. $4-6$ of Chen \& Ratra (2011b). We emphasize, however, that this effect is prominent only in some parts of the parameter spaces.

\section{Conclusion}

In summary, the results of a joint analysis of the $H(z)$, BAO, and SNIa data are very consistent with the predictions of a spatially-flat cosmological model with energy budget dominated by a time-independent cosmological constant, the standard $\Lambda$ CDM model. However, the data are not yet good enough to strongly rule out slowly-evolving dark energy density. More, and better quality, data are needed to discriminate between constant and slowly-evolving dark energy density.

It is probably quite significant that current $H(z)$ data constraints are almost as restrictive as those from SNIa data. The acquisition of $H(z)$ data has been an interesting 

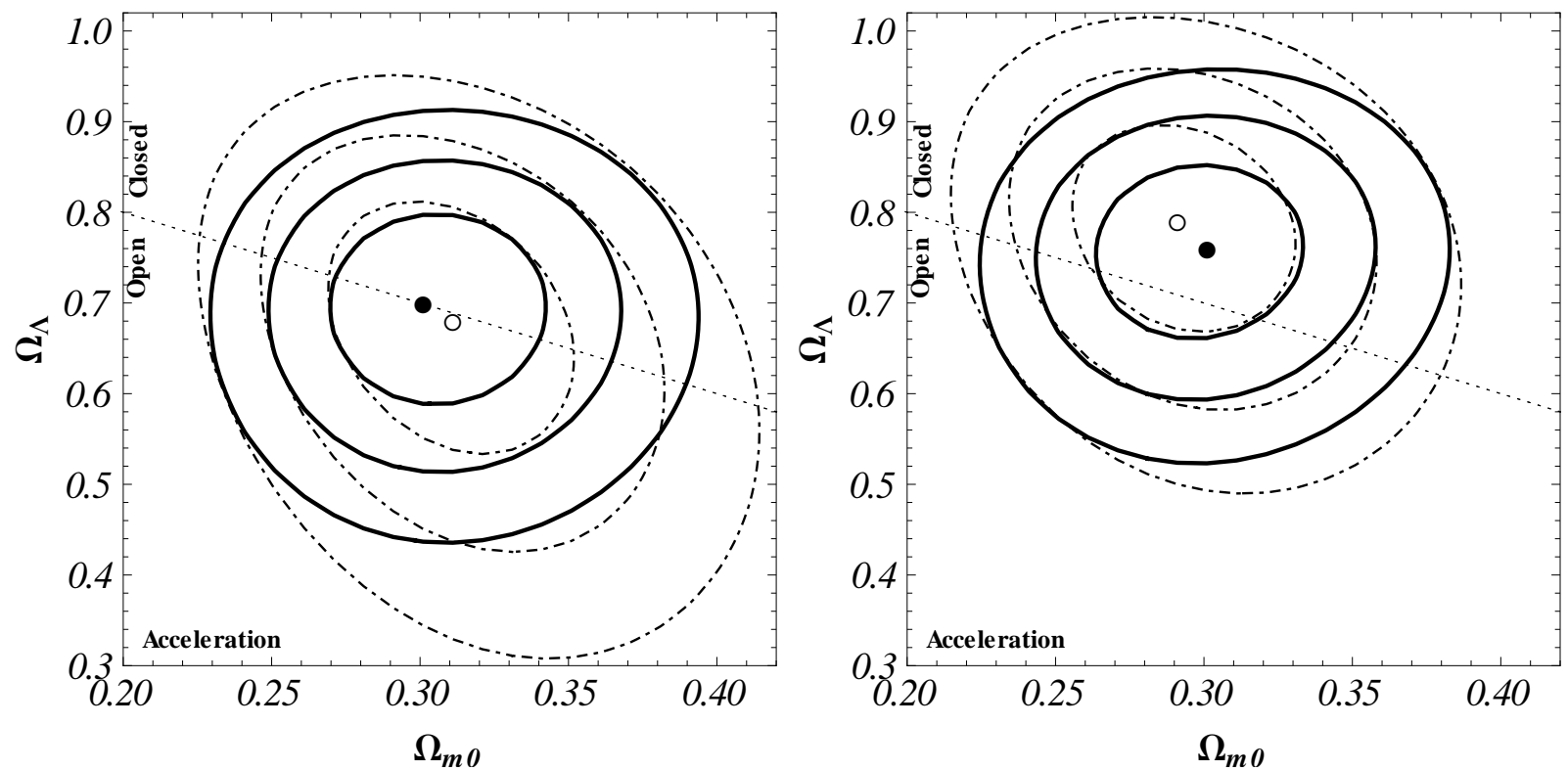

Fig. 9.- Thick solid (dot-dashed) lines are 1, 2, and $3 \sigma$ constraint contours for the $\Lambda$ CDM model from a joint analysis of the BAO and $H(z)$ data, with (without) the SNIa data. The full (empty) circle marks the best-fit point determined from the joint analysis with (without) the SNIa data. The dotted sloping line corresponds to spatially-flat $\Lambda$ CDM models. In the left panel we use the $H_{0}=68 \pm 2.8 \mathrm{~km} \mathrm{~s}^{-1} \mathrm{Mpc}^{-1}$ prior while the right panel is for the $H_{0}$ $=73.8 \pm 2.4 \mathrm{~km} \mathrm{~s}^{-1} \mathrm{Mpc}^{-1}$ case. For quantitative details see Table 3 .

backwater of cosmology for the last few years. We hope that our results will help promote more interest in this exciting area. Since the $H(z)$ technique has not been as much studied as, say, the SNIa apparent magnitude technique, a little more effort in the $H(z)$ area is likely to lead to very useful results.

We thank Chris Blake, Michele Moresco, Larry Weaver, and Shawn Westmoreland for useful discussions and helpful advice. We are grateful to the referee for a very detailed and prompt report that helped us improve our manuscript. This work was supported in part by DOE grant DEFG03-99EP41093 and NSF grant AST-1109275.

\section{REFERENCES}

Albrecht, A., et al. 2006, arXiv:astro-ph/0609591

Allen, S. W., et al. 2008, MNRAS, 383, 879 

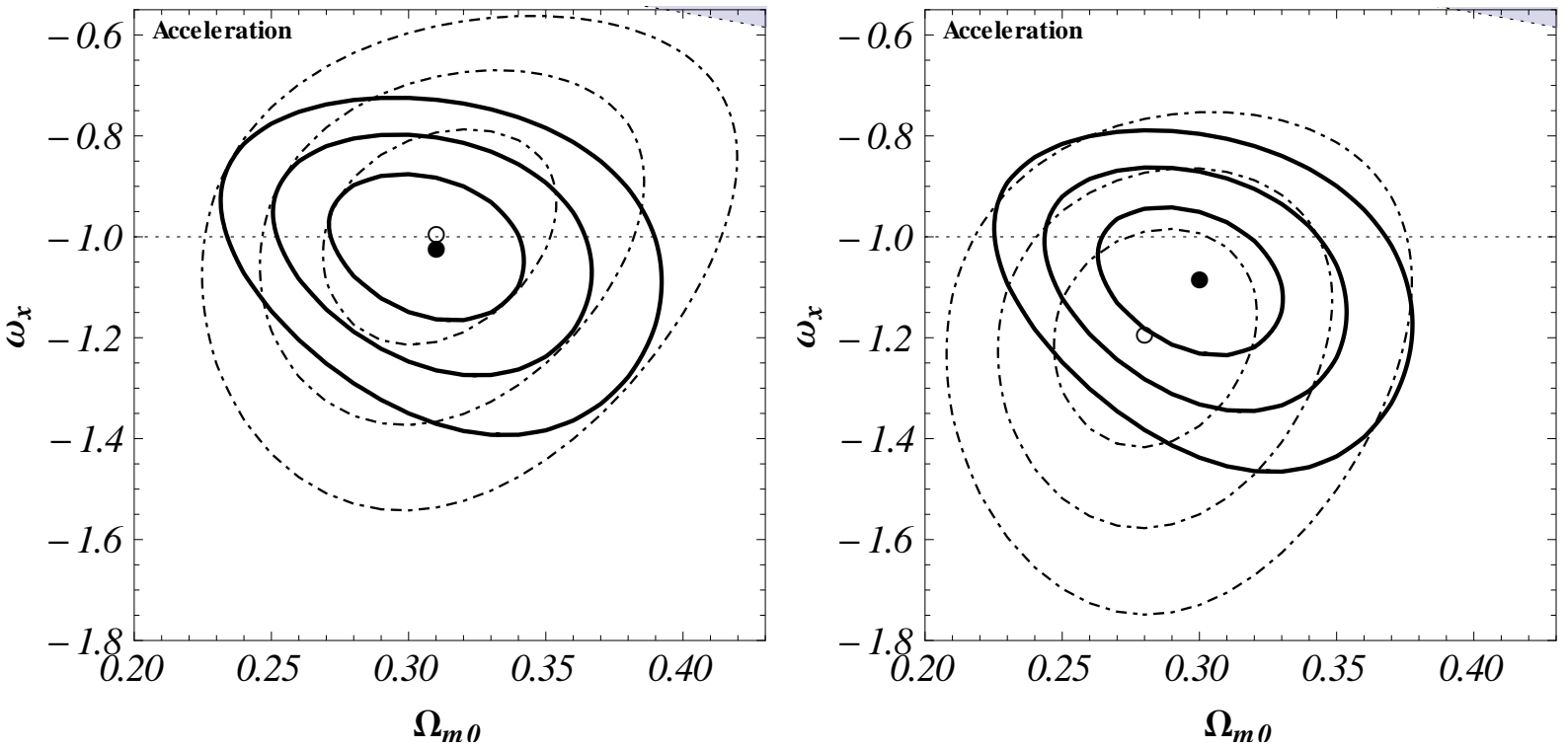

Fig. 10.- Thick solid (dot-dashed) lines are 1, 2, and $3 \sigma$ constraint contours for the XCDM parametrization from a joint analysis of the $\mathrm{BAO}$ and $H(z)$ data, with (without) the SNIa data. The full (empty) circle marks the best-fit point determined from the joint analysis with (without) the SNIa data. The dotted horizontal line at $\omega_{\mathrm{X}}=-1$ corresponds to spatially-flat $\Lambda \mathrm{CDM}$ models. In the left panel we use the $H_{0}=68 \pm 2.8 \mathrm{~km} \mathrm{~s}^{-1} \mathrm{Mpc}^{-1}$ prior while the right panel is for the $H_{0}=73.8 \pm 2.4 \mathrm{~km} \mathrm{~s}^{-1} \mathrm{Mpc}^{-1}$ case. The shaded area in the upper right corners are the region of decelerating expansion. For quantitative details see Table 3.

Barreira, A., \& Avelino, P. P. 2011, Phys. Rev. D, 84, 083521

Basilakos, S., et al. 2012, Astropart. Phys., 36, 7

Bass, S. D. 2011, J. Phys. G, 38, 043201

Basse, T., et al. 2012, arXiv:1205.0548 [astro-ph.CO]

Bauer, F., Solà, J., \& Štefančič, H. 2011, Mod. Phys. Lett. A, 26, 2559

Beutler, F., et al. 2011, MNRAS, 416, 3077

Biesiada, M., Piórkowska, A., \& Malec, B. 2010, MNRAS, 406, 1055

Bilicki, M., \& Seikel, M. 2012, MNRAS, 425, 1664

Blake, C., et al. 2011, MNRAS, 418, 1707

Bolotin, Yu. L., Lemets, O. A., \& Yerokhin, D. A. 2011, arXiv:1108.0203 [astro-ph.CO] 

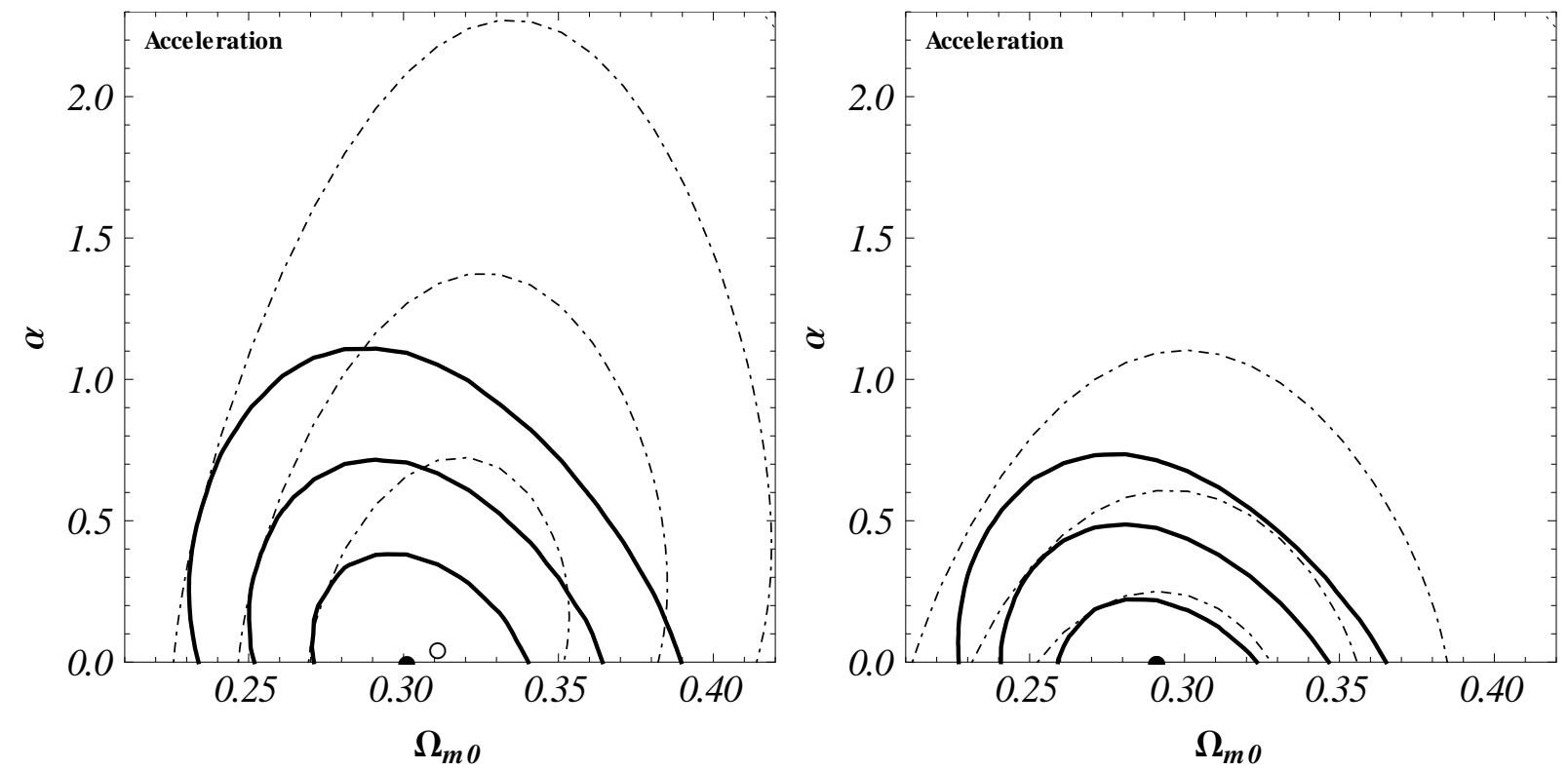

Fig. 11. - Thick solid (dot-dashed) lines are 1, 2, and $3 \sigma$ constraint contours for the $\phi$ CDM model from a joint analysis of the $H(z)$ and BAO data, with (without) the SNIa data. The full (empty) circle marks the best-fit point determined from the joint analysis with (without) the SNIa data. The $\alpha=0$ horizontal axes correspond to spatially-flat $\Lambda$ CDM models. In the left panel we use the $H_{0}=68 \pm 2.8 \mathrm{~km} \mathrm{~s}^{-1} \mathrm{Mpc}^{-1}$ prior while the right panel is for the $H_{0}=73.8 \pm 2.4 \mathrm{~km} \mathrm{~s}^{-1} \mathrm{Mpc}^{-1}$ case. For quantitative details see Table 3.

Bonamente, M., et al. 2006, ApJ, 647, 25

Brax, P., \& Davis, A.-C. 2012, Phys. Lett. B, 707, 1

Busti, V. C., Santos, R. C., \& Lima, J. A. S. 2012, Phys. Rev. D, 85, 103503

Cai, R.-G., at al. 2012, Phys. Rev. D, 86, 023511

Calabrese, E., et al. 2012, Phys. Rev. D, 86, 043520

Campanelli, L., et al. 2012, Eur. Phys. J. C, 72, 2218

Capozziello, S., \& De Laurentis, M. 2011, Phys. Rept., 509, 167

Chae, K.-H., et al. 2004, ApJ, 607, L71

Chen, G., Gott, J. R., \& Ratra, B. 2003, PASP, 115, 1269

Chen, G., \& Ratra, B. 2003, PASP, 115, 1143 


\begin{tabular}{cccc}
\hline \hline Model and prior & SNIa+BAO & $H(z)+\mathrm{BAO}$ & $H(z)+\mathrm{SNIa}+\mathrm{BAO}$ \\
\hline$\Lambda \mathrm{CDM}$ & $0.25<\Omega_{m 0}<0.36$ & $0.25<\Omega_{m 0}<0.36$ & $0.26<\Omega_{m 0}<0.36$ \\
$h=0.68 \pm 0.028$ & $0.53<\Omega_{\Lambda}<0.89$ & $0.45<\Omega_{\Lambda}<0.85$ & $0.55<\Omega_{\Lambda}<0.83$ \\
\hline$\Lambda \mathrm{CDM}$ & $0.25<\Omega_{m 0}<0.36$ & $0.23<\Omega_{m 0}<0.38$ & $0.25<\Omega_{m 0}<0.35$ \\
$h=0.738 \pm 0.024$ & $0.53<\Omega_{\Lambda}<0.89$ & $0.60<\Omega_{\Lambda}<0.92$ & $0.62<\Omega_{\Lambda}<0.88$ \\
\hline $\mathrm{XCDM}$ & $0.30<\Omega_{m 0}<0.38$ & $0.26<\Omega_{m 0}<0.37$ & $0.29<\Omega_{m 0}<0.37$ \\
$h=0.68 \pm 0.028$ & $-1.18<\omega_{\mathrm{X}}<-0.78$ & $-1.32<\omega_{\mathrm{X}}<-0.73$ & $-1.14<\omega_{\mathrm{X}}<-0.78$ \\
\hline $\mathrm{XCDM}$ & $0.30<\Omega_{m 0}<0.38$ & $0.24<\Omega_{m 0}<0.35$ & $0.27<\Omega_{m 0}<0.35$ \\
$h=0.738 \pm 0.024$ & $-1.18<\omega_{\mathrm{X}}<-0.78$ & $-1.42<\omega_{\mathrm{X}}<-0.88$ & $-1.22<\omega_{\mathrm{X}}<-0.86$ \\
\hline$\phi \mathrm{CDM}$ & $0.25<\Omega_{m 0}<0.35$ & $0.25<\Omega_{m 0}<0.36$ & $0.26<\Omega_{m 0}<0.35$ \\
$h=0.68 \pm 0.028$ & $0<\alpha<0.54$ & $0<\alpha<1.01$ & $0<\alpha<0.54$ \\
\hline$\phi \mathrm{CDM}$ & $0.25<\Omega_{m 0}<0.35$ & $0.23<\Omega_{m 0}<0.35$ & $0.25<\Omega_{m 0}<0.33$ \\
$h=0.738 \pm 0.024$ & $0<\alpha<0.54$ & $0<\alpha<0.57$ & $0<\alpha<0.35$ \\
\hline \hline
\end{tabular}

Table 4: Two standard deviation bounds on cosmological parameters using SNIa+BAO, $H(z)+\mathrm{BAO}$ and SNIa+BAO$+H(z)$ data, for 3 different models with two different $H_{0}$ priors.

Chen, G., \& Ratra, B. 2011a, PASP, 123, 1127

Chen, Y., \& Ratra, B. 2011b, Phys. Lett. B, 703, 406

Chen, Y., \& Ratra, B. 2012, A\&A, 543, A104

Chimento, L. P., Forte, M., \& Richarte, M. 2011, arXiv:1106.0781 [astro-ph.CO]

Dantas, M. A., et al. 2011, Phys. Lett. B, 699, 239

Davis, T. M., et al. 2007, ApJ, 666, 716

De Boni, C., et al. 2011, MNRAS, 415, 2758

Devi, N. C., Choudhury, T. R., \& Sen, A. A. 2011, arXiv:1112.0728 [astro-ph.CO]

Duan, X., Li, Y., \& Gao, C. 2011, arXiv:1111.3423 [astro-ph.CO]

Eisenstein, D. J., \& Hu, W. 1998, ApJ, 496, 605

Eisenstein, D. J., et al. 2005, ApJ, 633, 560

Ettori, S., et al. 2009, A\&A, 501, 61

Freedman, W. L., et al. 2012, ApJ, 758, 24 
Ganga, K., et al. 1997, ApJ, 484, 7

García-Bellido, J., et al. 2011, Phys. Rev. D, 84, 123504

Gaztañaga, E., Cabré, A., \& Hui, L. 2009, MNRAS, 399,1663

Gott, J. R., et al. 2001, ApJ, 549, 1

Granda, L. N., Torrente-Luján, E., \& Fernandez-Melgarejo, J. J. 2011, Eur. Phys. J. C, 71, 1704

Guerra, E. J., Daly, R. A., \& Wan, L. 2000, ApJ, 544, 659

Hollenstein, L., et al. 2012, Phys. Rev. D, 85, 124031

Jassal, H. K., Bagla, J. S., \& Padmanabhan, T. 2010, MNRAS, 405, 2639

Jennings, E., Baugh, C. M., \& Pascoli, S. 2012, MNRAS, 420, 1079

Jimenez, R. 2011, Fortschr. Phys., 59, 602

Jimenez, R., et al. 2003, ApJ, 593, 622

Komatsu, E., et al. 2011, ApJS, 192, 18

Kumar, S. 2012, MNRAS, 422, 2532

Lee, S., \& Ng, K.-W. 2007, Phys. Rev. D, 76, 043518

Li, M., et al. 2011a, Commun. Theor. Phys., 56, 525

Li, X.-D., et al. 2011b, J. Cosmology Astropart. Phys., 1107, 011

Lu, J., et al. 2011, Eur. Phys. J. C, 71, 1800

Mania, D., \& Ratra, B. 2012, Phys. Lett. B, 715, 9

Mehta, K. T., et al. 2012, arXiv:1202.0092 [astro-ph.CO]

Moresco, M., et al. 2012a, J. Cosmology Astropart. Phys., 1208, 006

Moresco, M., et al. 2012b, J. Cosmology Astropart. Phys., 1207, 053

Mortonson, M. J., Hu, W., \& Huterer, D. 2011, Phys. Rev. D, 83, 023015

Pan, N., et al. 2010, Class. Quantum Grav., 27, 155015 
Pavlov, A., Samushia, L., \& Ratra, B. 2012, ApJ, 760, 19

Peebles, P. J. E. 1984, ApJ, 284, 439

Peebles, P. J. E., \& Ratra, B. 1988, ApJ, 325, L17

Peebles, P. J. E., \& Ratra, B. 2003, Rev. Mod. Phys., 75, 559

Percival, W. J., et al. 2010, MNRAS, 401, 2148

Perivolaropoulos, L. 2010, J. Phys. Conf. Ser., 222, 012024

Plionis, M., et al. 2010, AIP Conf. Proc. 1241, 267

Plionis, M., et al. 2011, MNRAS, 416, 2981

Podariu, S., Nugent, P., \& Ratra, B. 2001a, ApJ, 553, 39

Podariu, S., et al. 2001b, ApJ, 559, 9

Podariu, S., \& Ratra, B. 2000, ApJ, 532, 109

Ratra, B. 1991, Phys. Rev. D, 43, 3802

Ratra, B., \& Peebles, P. J. E. 1988, Phys. Rev. D, 37, 3406

Ratra, B., \& Vogeley, M. S. 2008, PASP, 120, 235

Riess, A. G., et al. 2011, ApJ, 730, 119

Samushia, L., Chen, G., \& Ratra, B. 2007, arXiv:0706.1963 [astro-ph]

Samushia, L., et al. 2010, Phys. Lett. B, 693, 509

Samushia, L., \& Ratra, B. 2006, ApJ, 650, L5

Samushia, L., \& Ratra, B. 2008, ApJ, 680, L1

Samushia, L., \& Ratra, B. 2009, ApJ, 701, 1373

Samushia, L., \& Ratra, B. 2010, ApJ, 714, 1347

Samushia, L., et al. 2011, MNRAS, 410, 1993

Sartoris, B., et al. 2012, MNRAS, 423, 2503

Seikel, M., et al. 2012, Phys. Rev. D, 86, 083001 
Sen, A. A., \& Scherrer, R. J. 2008, Phys. Lett. B, 659, 457

Sheykhi, A., et al. 2012, Intl. J. Theo. Phys., 51, 1663

Simon, J., \& Verde,L., \& Jimenez, R. 2005, Phys. Rev. D, 71, 123001

Sorce, J. G., Tully, R. B., \& Courtois, H. M. 2012, ApJ, 758, L12

Starkman, G. D. 2011, Phil. Trans. Roy. Soc. Lond. A, 369, 5018

Stern, D., et al. 2010, JCAP 1002 (2010) 008

Sullivan, M., et al. 2011, ApJ, 737, 102

Suzuki, N., et al. 2012, ApJ, 746, 85

Tammann, G. A., \& Reindl, B. 2012, arXiv:1208.5054 [astro-ph.CO]

Tong, M., \& Noh, H. 2011, Eur. Phys. J. C, 71, 1586

Tonoiu, D., Caramete, A., \& Popa, L. A.. 2011, Rom. Rep. Phys., 63, 879

Tsujikawa, S. 2010, Lect. Notes Phys, 800, 99

van de Weygaert, R., et al. 2011, arXiv:1110.5528 [astro-ph.CO]

Wang, F. Y., \& Dai, J. G. 2011, A\&A, 536, A96

Wang, H., \& Zhang, T. J. 2012, ApJ, 748, 111

Wang, X., et al. 2012, J. Cosmology Astropart. Phys., 1211, 018

Wang, Y. 2012, MNRAS, 423, 3631

Weinberg, D. H., et al. 2012, arXiv:1201.2434 [astro-ph.CO]

Wilson, K. M., Chen, G., \& Ratra, B. 2006, Mod. Phys. Lett. A, 21, 2197

Zhang, C., et al. 2012, arXiv:1207.4541 [astro-ph.CO]

Zhang, Q.-J., \& Wu, Y.-L. 2010, J. Cosmology Astropart. Phys., 1008, 038

Ziaeepour, H. 2012, Phys. Rev. D, 86, 043503 